\title{
A Feeding Strategy in Inner L-Shape Ring Hot Rolling Process
}

\author{
Wen Meng, ${ }^{1,2}$ Feifan Wang, ${ }^{1}$ and Yanjin Guan ${ }^{2}$ \\ ${ }^{1}$ Key Laboratory for Advanced Materials Processing Technology, Ministry of Education, School of Materials Science and Engineering, \\ Tsinghua University, Beijing 100084, China \\ ${ }^{2}$ Key Laboratory for Liquid-Solid Structural Evolution and Processing of Materials, Ministry of Education, \\ School of Materials Science and Engineering, Shandong University, Jinan, Shandong 250061, China \\ Correspondence should be addressed to Yanjin Guan; guan_yanjin@sdu.edu.cn
}

Received 23 March 2017; Accepted 17 July 2017; Published 19 September 2017

Academic Editor: Yakov Strelniker

Copyright (C) 2017 Wen Meng et al. This is an open access article distributed under the Creative Commons Attribution License, which permits unrestricted use, distribution, and reproduction in any medium, provided the original work is properly cited.

In order to make the inner L-shape ring polling process with a closed die structure (ILRRCDS) on the top and bottom of the driven roll stable, at first, this paper established the mathematical model for ILRRCDS. Then, the plastic penetration and biting-in conditions for ILRRCDS were deduced based on plain ring rolling theory. Moreover, a feeding strategy that can realize a constant growth of the ring's outer radius was proposed and the reasonable value ranges of the feed rate of the mandrel were determined. The numerical simulation model for ILRRCDS is established based on ABAQUS software. Finally, the equivalent plastic strain (PEEQ) and temperature distributions of rolled ring were obtained. The results indicated that the proposed feeding strategy can realize a stable ILRRCDS. At the end of ILRRCDS, the PEEQ at the inner radius surface of the ring is maximum, the PEEQ at the outer radius surface of the ring takes the second place, and the PEEQ at the middle part of ring is minimum. With the increase of rolling time, the higher temperature zone of the rolled ring gradually moves from the center part of the ring to the "inner corner zone" of the ring.

\section{Introduction}

The inner L-shape ring hot rolling process with a closed die structure on the top and bottom of the driven roll (simplified as ILRRCDS) is an advanced plastic forming technology to manufacture seamless inner L-shape rings. It has many advantages in saving energy, saving materials, and decreasing noises. The rolled rings always have merits in comprehensive mechanical properties. Therefore, this ring rolling technology is widely applied in the fields of wind power, mining machinery, and naval construction, as reported by Allwood et al. [1].

With the growing needs of the community for profiled rings, profiled ring rolling technologies were increasingly used to manufacture profiled rings. However, compared with the ones in the rectangular ring rolling process, the metal flow laws are more complicated in profiled ring rolling processes. Recently, scholars studied several profiled ring rolling processes. Allwood et al. [2] analyzed a profiled ring rolling process in which the mandrel can move along both radial and axial directions and obtained the equivalent plastic strain distribution of the rolled ring. Based on ABAQUS software, Li et al. [3] investigated the effects of forming parameters on aluminum alloy $\mathrm{T}$-shape ring rolling process and pointed out that the increase of reduction is beneficial to maintain the deformation uniformities of the rolled ring. Kim et al. [4] studied a large-scale alloy steel profiled ring rolling process by simulation method based on MSC software and obtained the metal flow laws of the rolled ring. Hua et al. [5] proposed that there were three deformation behaviors in GCr15 alloy outer L-shape cross section cold ring rolling process and verified them by experiments. Yang et al. [6] pointed out that, with the increase of the reduction ratio of the ring's wall thickness, the growth of the ring's radius is accelerated during T-shape cold ring rolling processes. Lee et al. [7] investigated an inner L-shape ring rolling process with a profiled upper axial roll and obtained the equivalent plastic strain and temperature distributions of the rolled ring.

In the ring rolling process, the feeding strategy has significant effects on the stability of the rolling process and on both the dimensional accuracy and the comprehensive mechanical properties of the rolled ring. In recent years, in order to realize different aims, scholars designed corresponding feeding strategies in rectangular ring rolling processes. Kluge et al. [8] adopted a feeding strategy that could decrease 
the deformation degree of the materials located at the corners of the rolled rings' cross section to manufacture rectangular rings, and the feeding strategy could effectively control the equivalent plastic strain and temperature distributions of rolled Ti-6Al-4V alloy rectangular rings. Guo and Yang [9] proposed a feeding strategy that could make the ring's outer radius stably grow up in GH4169 steel radial-axial ring rolling process, determined the reasonable value ranges of the mandrel feed rate, and finally verified the correctness of the proposed feeding strategy by numerical simulation method. Kim et al. [10] decreased the maximum load of the rolling mill by optimizing the motions of rolls in radial-axial ring rolling process. Xu et al. [11] designed an effective feeding strategy to improve the mechanical properties of rolled rectangular rings based on 42CrMo processing diagram. Dish shaped rings were achieved by using the rolling strategy that causes the ring climbing proposed by Joachim S. et al. (2012). The controlling method of the guide rolls in double-groove rings rolling process was studied by Tian et al. [12]. Li et al. [13] investigated the influences of roll ratio on the inhomogeneity deformation degree of rolled double-groove rings by using simulation method. Giorleo et al. [14] verified the hot ring rolling industrial process by Deform-3D software and the experimental results matched the simulation ones well.

To sum up, scholars mainly studied the equivalent plastic strain distribution of T-shape and L-shape rings in radialaxial ring rolling processes. However, studies on the feeding strategy, plastic penetration, and biting-in conditions of rolled rings, controlling method of the motions of rolls, and the metal deformation laws were still insufficient.

Compared with the ones in the commonly seen rectangular ring rolling process, the metal flow laws of the inner L-shape ring in hot rolling process were more complicated. Unreasonable contacts between the guide rolls and ring may cause the instabilities in practical inner L-shape ring rolling process, and thus the mechanical properties and dimensional accuracy of rolled rings were worse. It is hard to choose the appropriate values of mandrel feed rate. Once the mandrel feed rate was wrongly chosen, there would be problems as follows: the rolled ring was blocked in the radial plastic deformation zone; the ring was hardly bitten into the gap between rolls; the top or bottom die of the driven roll was broken. In this situation, the further engineering applications of inner L-shape ring rolling technology would be restricted to some extent. Therefore, in order to solve the abovementioned problems, we must establish the mathematical model of the inner L-shape ring rolling process, determine the plastic penetration and biting-in conditions of the ring, select a reasonable feeding strategy, and study the metal flow laws in ILRRCDS.

\section{Theoretical Calculation}

Figure 1 shows the schematic diagram of ILRRCDS. In the rolling process, the driven roll rotates around its own fixed axis by a constant circumferential rate, $v_{D}$. The mandrel moves along the $-X$-axis direction by a set value. The ring's wall thickness gradually decreases while its diameter increases.
The guide rolls restrict the tilting of the ring and maintain the roundness of the rolled ring. The axial rolls restrict the vibration of the ring and keep the rolling process stable. In the rolling process, a similar closed die structure is made up of the driven roll, both the top and the bottom part of the driven roll, and the mandrel. This kind of die structure can restrict, to some extent, the metal flowing along the axial direction. Thus, the commonly seen fishtail defects can be effectively prevented.

ILRRCDS contains three forming stages: the first forming stage, the second forming stage, and the roundness correction stage. The first forming stage is from the moment that the mandrel starts to roll the ring to the moment that the feed amount of the mandrel just equals the step width of the Lshape ring (the step width of the L-shape ring is equal to $R_{M 1}-R_{M 2}$ ), as shown in Figures 1(a)-1(c). At the first forming stage, the ring's upper groove is rolled out by the upper part of the mandrel.

$R_{D}$ is the radius of the driven roll. $R_{M 1}$ is the upper radius of the mandrel. $R_{M 2}$ is the lower radius of the mandrel. $H_{M 1}$ is the height of the upper mandrel. $H_{M 2}$ is the height of the lower mandrel. $H$ is the height of the initial ring blank, $H=$ $H_{M 1}+H_{M 2} \cdot \gamma$ is the cone angle of axial roll. $R_{0}$ is the outer radius of the initial ring blank. $r_{0}$ is the inner radius of the initial ring blank. $R_{1}$ is the outer radius of the ring at the end of the first forming stage.

$r_{u 1}$ is the inner radius of the upper part of the ring (UPR) at the end of the first forming stage. $r_{l 1}$ is the inner radius of the lower part of the ring (LPR) at the end of the first forming stage. $R_{t}$ is the instantaneous outer radius of the ring at time $t$. $r_{u t}$ is the instantaneous inner radius of UPR at time $t . r_{l t}$ is the instantaneous inner radius of LPR at time t. $\Delta T_{1}$ is the time interval at the first forming stage.

The second forming stage is from the end of the first forming stage to the moment that the mandrel just right stops the feeding motion, as shown in Figures 1(c)-1(e). At the second forming stage, the ring's wall thickness gradually decreases while its diameter increases.

$R_{f}$ is the outer radius of the ring at the end of the second forming stage. $r_{u f}$ is the inner radius of UPR at the end of the second forming stage. $r_{l f}$ is the inner radius of LPR at the end of the second forming stage. $\Delta T_{2}$ is the time interval at the second forming stage.

The roundness correction stage is from the end of the second forming stage to the moment that the mandrel continues rolling another two revolutions. $\Delta T_{3}$ is the time interval at the roundness correction stage.

2.1. The Plastic Penetration and Biting-In Conditions in ILRRCDS. There are mainly three differences between the ILRRCDS and the plain rectangular ring rolling process (simplified as PRRR). First, the inner radii of UPR and LPR in ILRRCDS are different while the ring has no step in PRRR; second, the plastic penetration and biting-in conditions of the UPR are different from those of the LPR at a certain time in ILRRCDS; third, the change laws of the growth rate of the ring's outer radius in ILRRCDS are different from those in PRRR. 


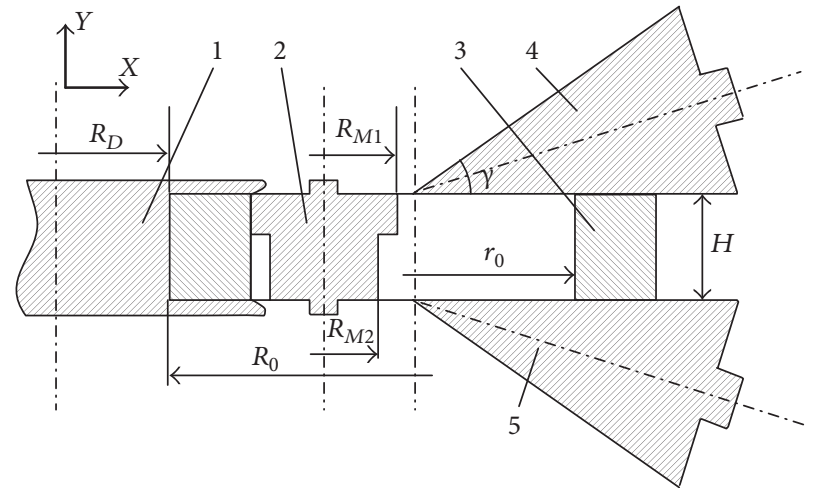

(1) Driven roll

(2) Mandrel

(3) Ring blank

(4) Upper axial roll

(5) Lower axial roll

(a) $t=0$

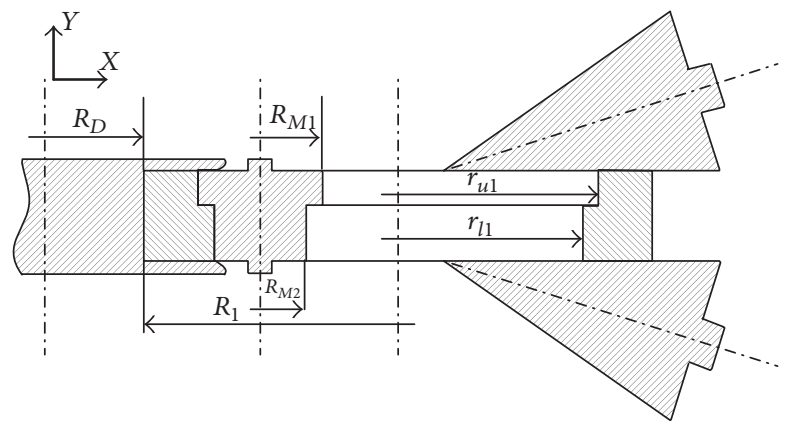

(c) $t=\Delta T_{1}$

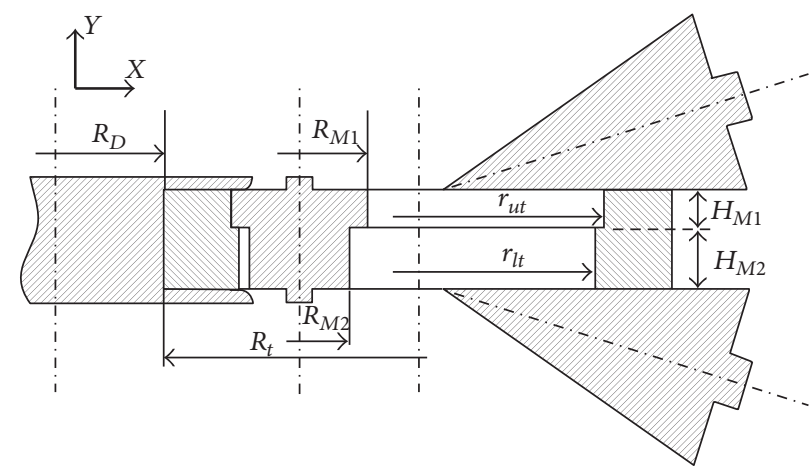

(b) $0<t<\Delta T_{1}$

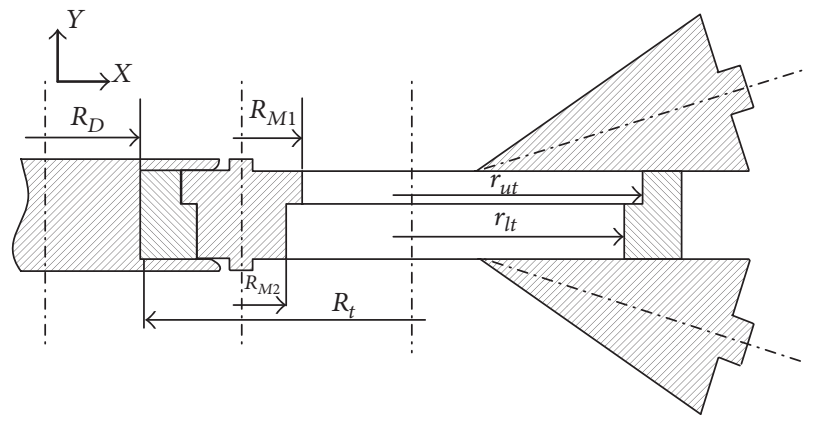

(d) $\Delta T_{1}<t<\Delta T_{1}+\Delta T_{2}$

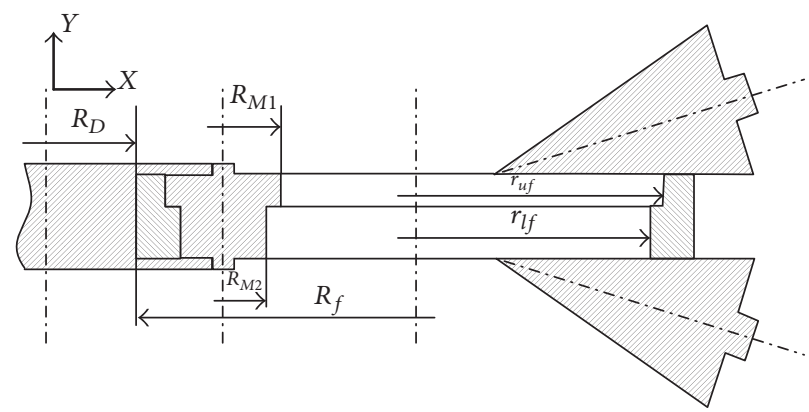

(e) $\Delta T_{1}+\Delta T_{2} \leq t \leq \Delta T_{1}+\Delta T_{2}+\Delta T_{3}$

FIGURE 1: Schematic diagram of the inner L-shape ring hot rolling process.

Besides, the UPR is similar to the rectangular cross section ring, and so is the LPR. That is to say, the metal plastic flow laws at a certain horizontal plane in ILRRCDS are similar to those in PRRR. Therefore, it is reasonable to use the plastic penetration and biting-in conditions of the rectangular ring in PRRR to analyze the plastic penetration and biting-in conditions of the L-shape ring in ILRRCDS.

This paper considered the similarity of the metal flow laws and the differences of the cross section of the rings between ILRRCDS and PRRR and developed the plain ring rolling theory to analyze the metal flow laws of the ring in ILRRCDS.

Figure 2 shows the metal deformation principle in ILRRCDS. In Figure 2, $\mathrm{O}_{1}, \mathrm{O}_{2}$, and $\mathrm{O}_{3}$ are the centers of the driven roll, the mandrel, and the inner L-shape ring, respectively. $L_{1}$ is the projected length of the contact circular arc $A B$ between UPR and driven roll on $Z$-axis. $L_{2}$ is the projected length of the contact circular arc $C D$ between LPR and mandrel on $Z$-axis. $\Delta h_{D}, \Delta h_{M 1}$, and $\Delta h_{M 2}$ are the feed amount per revolution of the driven roll, the upper part of the mandrel, and the lower part of the mandrel, respectively. $L_{1}^{\prime}$ is the projected length of the contact circular arc $A^{\prime} B^{\prime}$ between the LPR and driven roll on $Z$-axis. $L_{2}^{\prime}$ is the projected length of the contact circular arc $C^{\prime} D^{\prime}$ between the LPR and mandrel on $Z$-axis.

In Figure 2(a), according to plain ring rolling theory reported by Hua and Zhao [16], at the first forming stage of 


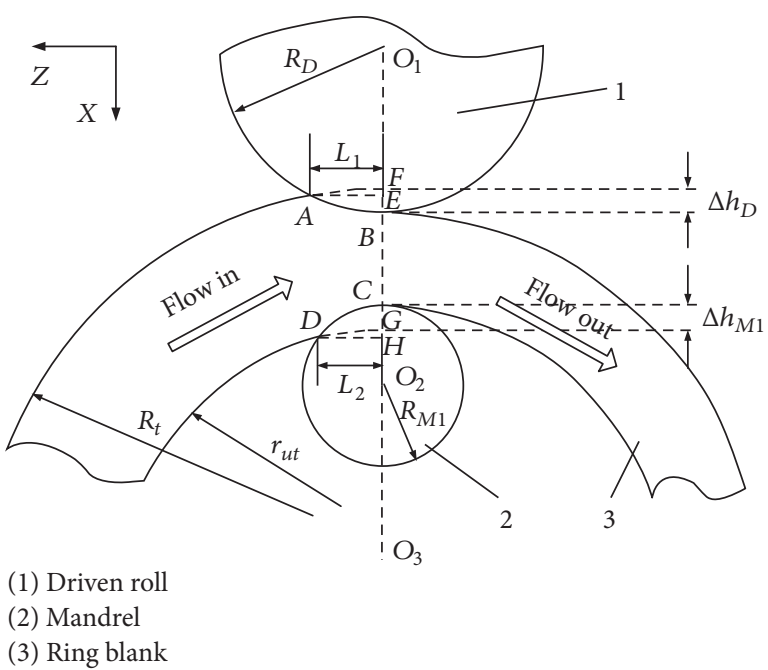

(a)

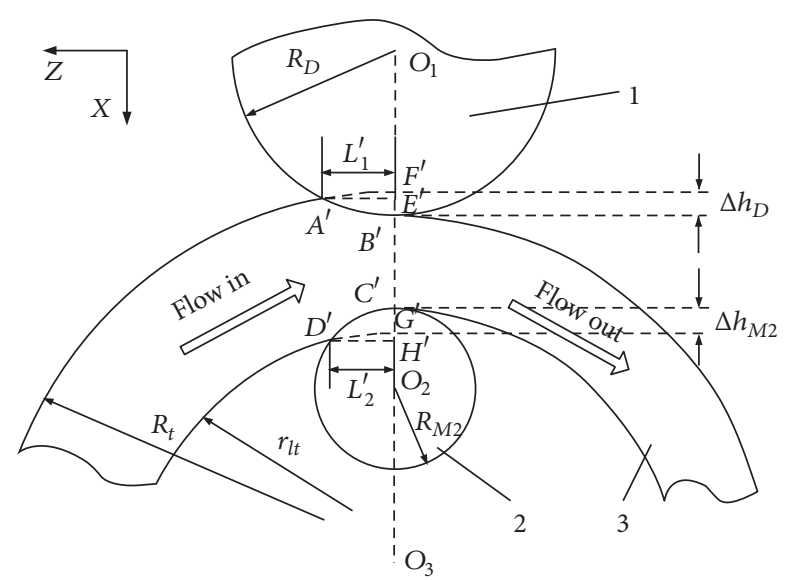

(b)

FIGURE 2: The metal deformation principle in ILRRCDS: (a) the metal deformation principle of the UPR in the whole ILRRCDS; (b) the metal deformation principle of the LPR at the second forming stage in ILRRCDS.

ILRRCDS, the plastic penetration and biting-in conditions of UPR should satisfy the following equations:

$$
\begin{aligned}
& \Delta h_{\min , u 1}=0.00665 \\
& \quad \times\left(R_{t}-r_{u t}\right)^{2}\left(\frac{1}{R_{D}}+\frac{1}{R_{M 1}}+\frac{1}{R_{t}}-\frac{1}{r_{u t}}\right), \\
& \Delta h_{\max , u 1} \\
& =\frac{2 \beta^{2}}{\left(1 / R_{D}+1 / R_{M 1}\right)^{2}}\left(\frac{1}{R_{D}}+\frac{1}{R_{M 1}}+\frac{1}{R_{t}}-\frac{1}{r_{u t}}\right), \\
& \Delta h_{\min , u 1} \leq \Delta h_{u 1} \leq \Delta h_{\max , u 1}, 0<t<\Delta T_{1},
\end{aligned}
$$

where $\Delta h_{u 1}$ is the feed amount per revolution of UPR at time $t$ at the first forming stage of ILRRCDS, $\Delta h_{u 1}=$ $\Delta h_{D}+\Delta h_{M 1} . \Delta h_{D}(l, t)$ and $\Delta h_{M}(l, t)$ are the feed amount per revolution of the driven roll and the upper part of the mandrel at time $t$, respectively. $\Delta h_{\min , u 1}$ and $\Delta h_{\max , u 1}$ are the permitted minimum and maximum of $\Delta h_{u 1}$, respectively. $\beta$ is the friction angle between the inner L-shape ring and rolls, $\beta=\arctan \mu . \mu$ is the friction factor between the inner Lshape ring and rolls.

In Figure 2(b), at the second forming stage in ILRRCDS, the plastic penetration and biting-in conditions of UPR should satisfy the following equations:

$$
\begin{aligned}
& \Delta h_{\min , u 2}=0.00665 \\
& \quad \times\left(R_{t}-r_{u t}\right)^{2}\left(\frac{1}{R_{D}}+\frac{1}{R_{M 1}}+\frac{1}{R_{t}}-\frac{1}{r_{u t}}\right) \\
& \Delta h_{\max , u 2} \\
& \quad=\frac{2 \beta^{2}}{\left(1 / R_{D}+1 / R_{M 1}\right)^{2}}\left(\frac{1}{R_{D}}+\frac{1}{R_{M 1}}+\frac{1}{R_{t}}-\frac{1}{r_{u t}}\right), \\
& \quad \Delta h_{\min , u 2} \leq \Delta h_{u 2} \leq \Delta h_{\max , u 2}, \Delta T_{1}<t<\Delta T_{1}+\Delta T_{2},
\end{aligned}
$$

where $\Delta h_{u 2}$ is the feed amount per revolution of UPR at time $t$ at the second forming stage in ILRRCDS, $\Delta h_{u 2}=\Delta h_{D}+$ $\Delta h_{M 1} . \Delta h_{\min , u 2}$ and $\Delta h_{\max , u 2}$ are the permitted minimum and maximum values of $\Delta h_{u 2}$, respectively.

According to Hua and Zhao [16] and by assuming $L_{1}=$ $L_{2}=L$, we can obtain

$$
\Delta h_{u 2}=\frac{L^{2}}{2}\left(\frac{1}{R_{D}}+\frac{1}{R_{M 1}}+\frac{1}{R_{t}}-\frac{1}{r_{u t}}\right) .
$$

At the second forming stage in ILRRCDS, the plastic penetration and biting-in conditions of LPR should satisfy the following equations:

$$
\begin{aligned}
& \Delta h_{\min , l 2}=0.00665 \\
& \quad \times\left(R_{t}-r_{l t}\right)^{2}\left(\frac{1}{R_{D}}+\frac{1}{R_{M 2}}+\frac{1}{R_{t}}-\frac{1}{r_{l t}}\right), \\
& \Delta h_{\max , l 2} \\
& \quad=\frac{2 \beta^{2}}{\left(1 / R_{D}+1 / R_{M 2}\right)^{2}}\left(\frac{1}{R_{D}}+\frac{1}{R_{M 2}}+\frac{1}{R_{t}}-\frac{1}{r_{l t}}\right), \\
& \Delta h_{\min , l 2} \leq \Delta h_{l 2} \leq \Delta h_{\max , l 2}, \Delta T_{1}<t<\Delta T_{1}+\Delta T_{2},
\end{aligned}
$$

where $\Delta h_{l 2}$ is the feed amount per revolution of UPR at time $t$ at the second forming stage in ILRRCDS, $\Delta h_{l 2}=\Delta h_{D}+$ $\Delta h_{M 2} . \Delta h_{\min , l 2}$ and $\Delta h_{\max , l 2}$ are the permitted minimum and maximum values of $\Delta h_{l 2}$, respectively. obtain

According to [16] and by assuming $L_{1}^{\prime}=L_{2}^{\prime}=L^{\prime}$, we

$$
\Delta h_{u 2}=\frac{L^{\prime 2}}{2}\left(\frac{1}{R_{D}}+\frac{1}{R_{M 2}}+\frac{1}{R_{t}}-\frac{1}{r_{u t}}\right),
$$

where $\Delta h_{2}$ is named as the feed amount per revolution of the ring at time $t$ at the second forming stage in ILRRCDS; we get

$$
\Delta h_{2}=\Delta h_{u 2}=\Delta h_{l 2} \text {. }
$$


According to (6) and (10), the following equation can be obtained:

$$
\Delta h_{2, \min } \leq \Delta h_{2} \leq \Delta h_{2, \max }
$$

where $\Delta h_{2, \min }=\max \left\{\Delta h_{\min , u 2}, \Delta h_{\min , l_{2}}\right\}, \Delta h_{2, \max }=$ $\min \left\{\Delta h_{\max , u 2}, \Delta h_{\max , l_{2}}\right\}$.

In conclusion, the plastic penetration and biting-in conditions of both UPR and LPR should be satisfied simultaneously in ILRRCDS.

2.2. The Feeding Strategy in ILRRCDS. Assuming that the mandrel moved towards the driven roll by a constant rate in ILRRCDS, the inner L-shape ring will grow faster. In this situation, neither the guide rolls nor the axial rolls can control the deformation of the ring; therefore, the ring may vacillate and vibrate. This may lead to the decrease of the mechanical properties and the dimensional accuracy of rolled rings.

Recently, Guo and Yang [9], Pan [17], and Lee and Kim [18] studied the feeding strategies in rectangular ring rolling processes, respectively. They pointed out that the feeding strategy that can achieve a constant ring's outer radius growth rate may maintain the stability of the rolling processes. Lee and Kim [18] verified the feeding strategy mentioned above by using FE simulation and experimental methods. The mandrel feeding strategy deduced by them can only be used in radial-axial rectangular ring rolling processes, but it cannot be directly used in ILRRCDS.

Therefore, in order to realize a stable ILRRCDS, this paper proposed a feeding strategy and deduced the reasonable value ranges of the mandrel feed rate.

The proposed feeding strategy is as follows: first, a suitable constant mandrel feed rate $v_{m 1}$ is adopted at the first forming stage in ILRRCDS, because only the UPR is deformed; second, the feeding strategy that can achieve a constant ring's outer radius growth rate is adopted at the second forming stage in ILRRCDS; third, the mandrel continues rolling another two revolutions at the roundness correction stage to make the ring round.

By assuming that the outer radii of both UPR and LPR grow up simultaneously, according to the principle of volume constancy, in ILRRCDS, the ring's outer radius at time $t$ can be expressed as follows:

$$
R_{1}=\frac{V_{0}+\pi\left(H_{M 1} b_{u 1}^{2}+H_{M 2} b_{l 1}^{2}\right)}{2 \pi\left[H_{M 1} b_{u 1}+H_{M 2} b_{l 1}\right]}
$$

where $V_{0}$ is the volume of the initial ring blank, $V_{0}=\pi\left(H_{M 1}+\right.$ $\left.H_{M 2}\right)\left(R_{0}^{2}-r_{0}^{2}\right)$. The wall thickness of LPR, $b_{l t}$, is constant at the first forming stage in ILRRCDS. The thickness of UPR, $b_{u t}$, gradually decreases at the first forming stage in ILRRCDS; $b_{u t}=b_{0}-\int v_{m 1} d t,\left(0 \leq t \leq \Delta T_{1}\right)$.

At the end of the second forming stage in ILRRCDS, the ring's outer radius can be expressed as follows:

$$
R_{f}=\frac{V_{0}+\pi\left(H_{M 1} b_{u f}^{2}+H_{M 2} b_{l f}^{2}\right)}{2 \pi\left[H_{M 1} b_{u f}+H_{M 2} b_{l f}\right]}
$$

where $b_{u f}$ is the wall thickness of the UPR at the end of the second forming stage in ILRRCDS, $b_{u f}=R_{f}-r_{u f} . r_{u f}$ is the inner radius of the UPR at the end of the second forming stage in ILRRCDS. $b_{l f}$ is the wall thickness of the LPR at the end of the second forming stage in ILRRCDS, $b_{l f}=R_{f}-r_{l f} . r_{l f}$ is the inner radius of the LPR at the end of the second forming stage in ILRRCDS.

On the one hand, the instantaneous outer radius of the ring, $R_{t}$, can be expressed as follows:

$$
R_{t}= \begin{cases}R_{0}+\frac{\left(R_{1}-R_{0}\right) v_{m 1} t}{R_{M 1}-R_{M 2}}, & 0 \leq t<\Delta T_{1} \\ R_{1}+v_{R 2} t, & \Delta T_{1} \leq t \leq \Delta T_{1}+\Delta T_{2}\end{cases}
$$

where $v_{m 1}$ is the constant mandrel feed rate at the first forming stage; $v_{R 2}$ is the constant growth rate of the ring's outer radius at the second forming stage; $t$ is time; $\Delta T_{1}$ is the time interval of the first forming stage, $\Delta T_{1}=\left(R_{M 1}-\right.$ $\left.R_{M 2}\right) / v_{m 1} ; \Delta T_{2}$ is the time interval of the second forming stage, $\Delta T_{2}=\left(R_{f}-R_{1}\right) / v_{R 2}$.

On the other hand, the instantaneous outer radius of the ring, $R_{t}$, can be also expressed as

$$
R_{t}=\frac{V_{0}+\pi\left[H_{M 1}\left(b_{l t}-R_{M 1}+R_{M 2}\right)^{2}+H_{M 2} b_{l t}^{2}\right]}{2 \pi\left[H_{M 1}\left(b_{l t}-R_{M 1}+R_{M 2}\right)+H_{M 2} b_{l t}\right]},
$$

where $b_{l t}$ is the instantaneous wall thickness of LPR, $b_{l t}=b_{0}$ $\int v_{t} d t,\left(\Delta T_{1} \leq t \leq \Delta T_{1}+\Delta T_{2}\right) . v_{t}$ is the instantaneous mandrel feed rate. The instantaneous wall thickness of UPR, $b_{u t}$, can be calculated by $b_{u t}=b_{0}-\int v_{t} d t-v_{m 1} \Delta T_{1},\left(\Delta T_{1} \leq t \leq \Delta T_{1}+\Delta T_{2}\right)$.

Taking the derivative of (17) with respect to time $t$, the instantaneous mandrel feed rate, $v_{t}$, can be obtained:

$$
v_{t}=\frac{2 \pi\left[H_{M 1}\left(b_{l t}-R_{M 1}+R_{M 2}\right)+H_{M 2} b_{l t}\right]^{2}}{H\left[V_{0}+\pi H_{M 1}\left(b_{l t}-R_{M 1}+R_{M 2}\right)^{2}+\pi H_{M 2} b_{l t}^{2}\right]-2 \pi\left[H_{M 1}\left(b_{l t}-R_{M 1}+R_{M 2}\right)+H_{M 2} b_{l t}\right]^{2}} v_{R 2} .
$$

2.3. The Reasonable Value Ranges of the Mandrel Feed Rate. If we ignore the sliding motion between the rolls and ring in ILRRCDS at time $t$ at the first forming stage, the length of a circle where the ring's outer radius rotates is equal to the length where the radius of the driven roll rotates. Therefore, we have

$$
2 \pi R_{t}=v_{D} \Delta t_{1}, \quad\left(R_{0} \leq R_{t} \leq R_{1}, 0 \leq t<\Delta T_{1}\right),
$$


where $v_{D}$ is the peripheral velocity of the driven roll, $v_{D}=$ $R_{D} n_{D} ; n_{D}$ is the angular velocity of the driven roll. $\Delta t_{1}$ is the needed time for the ring to rotate a revolution.

The feed amount per revolution of the ring can be expressed as

$$
\Delta h_{u 1}=v_{m 1} \Delta t_{1} .
$$

According to (3), (19), and (20), we can get

$$
\frac{v_{D} \Delta h_{\min , u 1}}{2 \pi R_{t}} \leq v_{m 1} \leq \frac{v_{D} \Delta h_{\max , u 1}}{2 \pi R_{t}}, \quad\left(0 \leq t<\Delta T_{1}\right) .
$$

For convenience, we order $f_{1}(t)=v_{D} \Delta h_{\min , u 1} / 2 \pi R_{t}$, $g_{1}(t)=v_{D} \Delta h_{\max , u 1} / 2 \pi R_{t},\left(0 \leq t<\Delta T_{1}\right)$.

If we ignore the sliding motion between the rolls and ring in ILRRCDS at time $t$ at the second forming stage, the length of a circle where the ring's outer radius rotates is equal to the length where the radius of the driven roll rotates. Therefore, we have

$$
2 \pi R_{t}=v_{D} \Delta t_{2}, \quad\left(\Delta T_{1} \leq t \leq \Delta T_{1}+\Delta T_{2}\right),
$$

where $\Delta t_{2}$ is the time needed for the ring to rotate a circle.

The feed amount per revolution of the ring can be expressed as follows:

$$
\Delta h_{2} \approx v_{t} \Delta t_{2} .
$$

According to (13), (22), and (23), we can obtain

$$
\frac{v_{D} \Delta h_{2, \min }}{2 \pi R_{t}} \leq v_{t} \leq \frac{v_{D} \Delta h_{2, \max }}{2 \pi R_{t}}
$$

$$
\left(\Delta T_{1} \leq t \leq \Delta T_{1}+\Delta T_{2}\right) .
$$

For convenience, we set $f_{2}(t)=v_{D} \Delta h_{2, \min } / 2 \pi R_{t}, g_{2}(t)=$ $v_{D} \Delta h_{2, \max } / 2 \pi R_{t},\left(\Delta T_{1} \leq t \leq \Delta T_{1}+\Delta T_{2}\right)$.

According to (18), (23), and (24), the reasonable value ranges of $v_{R 2}$ can be expressed as follows:

$$
\begin{aligned}
& f_{2}(t) \\
& \cdot\left[\frac{H\left[V_{0}+\pi H_{M 1}\left(b_{l t}-R_{M 1}+R_{M 2}\right)^{2}+\pi H_{M 2} b_{l t}^{2}\right]}{2 \pi\left[H_{M 1}\left(b_{l t}-R_{M 1}+R_{M 2}\right)+H_{M 2} b_{l t}\right]^{2}}\right. \\
& -1] \leq v_{R 2} \leq g_{2}(t) \\
& \cdot\left[\frac{H\left[V_{0}+\pi H_{M 1}\left(b_{l t}-R_{M 1}+R_{M 2}\right)^{2}+\pi H_{M 2} b_{l t}^{2}\right]}{2 \pi\left[H_{M 1}\left(b_{l t}-R_{M 1}+R_{M 2}\right)+H_{M 2} b_{l t}\right]^{2}}\right. \\
& \quad-1] .
\end{aligned}
$$

\section{The FE Modeling of ILRRCDS}

According to the feeding strategy established in Section 2, the FE model of ILRRCDS was set up based on ABAQUS/Explicit software, as shown in Figure 3. The key FE modeling techniques are expressed as follows.

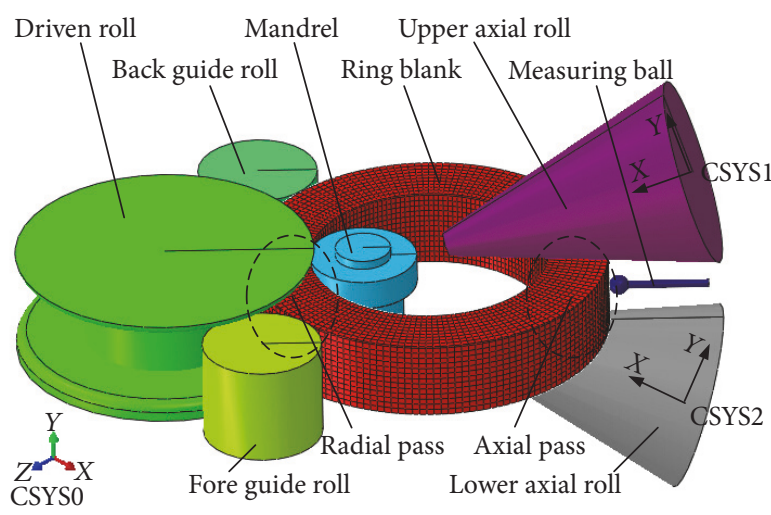

FIGURE 3: FE model for ILRRCDS.

TABLE 1: Values of coefficients in the constitutive model for S355NL [15].

\begin{tabular}{lc}
\hline Coefficients & Values \\
\hline $\mathrm{Q}\left(\mathrm{kJ} \cdot \mathrm{mol}^{-1}\right)$ & 450.10 \\
$R\left(\mathrm{~J} \cdot(\mathrm{mol} \cdot \mathrm{K})^{-1}\right)$ & 8.314 \\
$A$ & $5.70 \times 10^{15}$ \\
$\alpha$ & 0.015 \\
$n$ & 5.71 \\
\hline
\end{tabular}

TABLE 2: Physical property parameters and values for S355NL [7].

\begin{tabular}{lc}
\hline Parameters & Values \\
\hline Density $\left(\mathrm{kg} / \mathrm{m}^{3}\right)$ & 7850 \\
Poisson's ratio & 0.3 \\
Young's modulus $(\mathrm{GPa})$ & 210 \\
Specific heat $\left(\mathrm{J} /\left(\mathrm{kg}{ }^{\circ} \mathrm{C}\right)\right)$ & 450 \\
Linear expansion coefficient $\left({ }^{\circ} \mathrm{C}^{-1}\right)$ & $1.5 \times 10^{-5}$ \\
Contact thermal conduction $\left(\mathrm{W} /\left(\mathrm{m}^{2}{ }^{\circ} \mathrm{C}\right)\right)$ & 10000 \\
Coefficient of thermal convection $\left(\mathrm{W} /\left(\mathrm{m}^{2}{ }^{\circ} \mathrm{C}\right)\right)$ & 20 \\
Thermal emissivity $\left(\mathrm{Ns}^{-1} \mathrm{~mm}^{-1}{ }^{\circ} \mathrm{C}^{-4}\right)$ & 0.6 \\
\hline
\end{tabular}

3.1. Material Constitutive Model. The material of the ring blank is S355NL (Chinese standard is Q345E). Its constitutive model within the forging temperature range $\left(900^{\circ} \mathrm{C} \sim 1250^{\circ} \mathrm{C}\right)$ established by Li et al. [15] is adopted; that is,

$$
Z=\dot{\varepsilon} \exp \left(\frac{Q}{(R T)}\right)=A[\sinh \alpha \sigma]^{n},
$$

where $Z$ is the Zener-Hollomon parameter. $\dot{\varepsilon}$ is the strain rate. $Q$ is the deformation activation energy for S355NL. $R$ is the universal gas constant. $T$ is the absolute temperature. $A$ is a constant. $\alpha$ is the optimization factor of the material. $n$ is the working hardening exponent of the material. The values of the coefficients in the above equations are shown in Table 1. S355NL steel's density, Poisson's ratio, Young's modulus, specific heat, linear expansion coefficient, contact thermal conduction, coefficient of thermal convection, and thermal emissivity are listed in Table 2 as reported by Lee et al. [7]. 


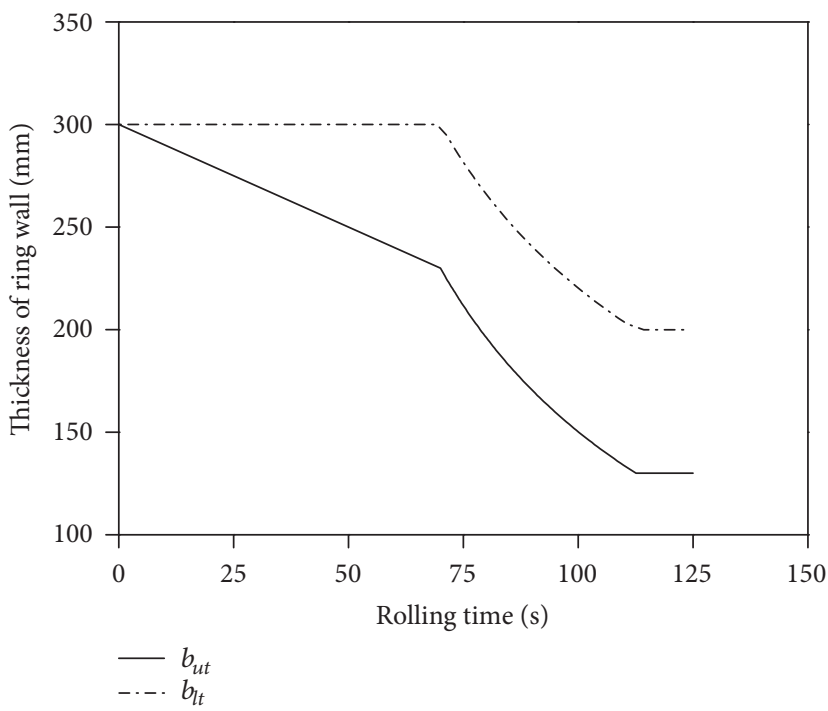

(a)

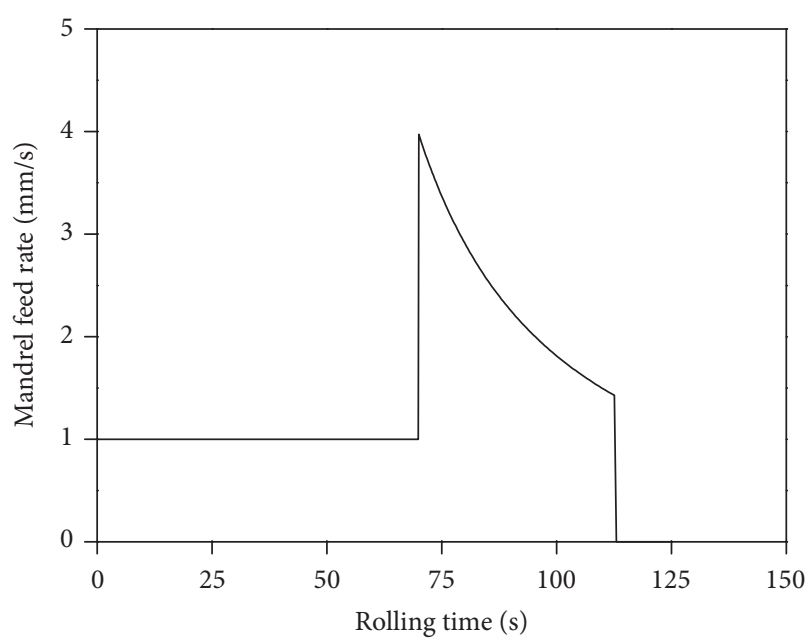

(b)

FIGURE 4: (a) The changing curves of the thickness of the ring with rolling time; (b) the changing curve of mandrel feed rate with rolling time.

3.2. CAE Modeling. The ring blank is defined as a threedimensional deformable body. The coupled thermodisplacement element with eight nodes and six faces (C3D8RT) was adopted to mesh the ring blank according to ABAQUS 6.14 User's Manual [19]. The number of finite element meshes is approximately 10,300. Reduction integration and ALE remeshing technology are also adopted to avoid the nonconvergence of the finite element numerical solution caused by severely distorted meshes as declared by Zhou et al. [20]. Hourglass control is employed to avoid zero energy modes. In order to reduce the calculated time, the mass scaling technology and dynamic explicit algorithm are employed. The mass scaling factor is set as 50 . The time scaling factor is set as 1. Six pairs of contact relationships between the ring and the rolls, such as the driven roll, mandrel, fore guide roll, back guide roll, upper axial roll, and lower axial roll, are defined. The frictional conditions and thermal conditions such as thermal conduction, thermal convection, and thermal radiation are considered in the FE model. According to the investigation by Shiro et al. [21], the friction factor on the interfaces between the ring and the roll can be set as 0.26. The temperatures of all rolls are set as $200^{\circ} \mathrm{C}$. The initial temperature of blank is set as $1200^{\circ} \mathrm{C}$.

3.3. The Motions Control of Rolls. In Figure 3, the ring blank and all rolls are assembled under the global coordinate system, CSYSO. All rolls are defined as rigid bodies. Each roll has its own reference point. The rotational and translational motions of rolls can be controlled by setting the motion conditions of their own corresponding reference points under their own coordinate system. The coordinate system of the driven roll, guide rolls, and the mandrel is CSYS0. The local coordinate system of the upper axial roll is CSYS1. The local coordinate system of the lower axial roll is CSYS2. The mandrel moves along the $-X$-axis direction.
The $X$ coordinate of mandrel at time $t, x_{t}$, can be obtained according to the equation $x_{t}=x_{0}-\int v_{t}^{\prime} d t$, where $x_{0}$ is the $X$ coordinate of mandrel at the initial time. The reference points of guide rolls move in $X O Z$ plane, while the reference points of axial rolls move in XOY plane. The movement locus (displacement-time relationship curves) of guide rolls and axial rolls can be obtained from the investigation reported by Pan [17].

\section{Validation of the FE Model for ILRRCDS}

The ILRRCDS experiments were carried out in Ireate Company. The material of ring blank is S355NL. The experimental forming parameters are shown in Table 3. According to (21) and (25), the reasonable value ranges of $v_{m 1}$ and $v_{R 2}$ are $0.76 \mathrm{~mm} / \mathrm{s} \leq v_{m 1} \leq 4.46 \mathrm{~mm} / \mathrm{s}$ and $2.53 \mathrm{~mm} / \mathrm{s} \leq$ $v_{R 2} \leq 14.91 \mathrm{~mm} / \mathrm{s}$, respectively. In order to satisfy the plastic penetration and biting-in conditions of the ring in the whole ILRRCDS, we selected $v_{m 1}=1 \mathrm{~mm} / \mathrm{s}$ and $v_{R 2}=$ $10 \mathrm{~mm} / \mathrm{s}$. According to (14) and (17), the changing curves of the thickness of the ring with rolling time can be calculated as shown in Figure 4(a). The height of the ring is constant in the established mathematical model for inner L-shape ring hot rolling process. The wall thickness of UPR, $b_{u t}$, gradually decreases in the first and the second forming stages. The wall thickness of LPR, $b_{l t}$, gradually decreases in the second forming stage. According to (18), the changing curve of mandrel feed rate with rolling time can be calculated as shown in Figure 4(b).

Figure 5(a) shows the rolling mill in ILRRCDS. Figure 5(b) shows the rolled inner L-shape ring. Both the outer radius and the inner radius of the ring can be measured online by measuring roll. At the end of the rolling process, the temperature values at different positions of the rolled ring were measured, as shown in Figure 6. Each measuring position 
TABLE 3: Forming parameters for experimental ILRRCDS.

\begin{tabular}{lc}
\hline Parameters & Values \\
\hline Radius of driven roll $(\mathrm{mm})$ & 425.00 \\
Radius of upper mandrel $(\mathrm{mm})$ & 270.00 \\
Radius of lower mandrel $(\mathrm{mm})$ & 200.00 \\
Height of upper mandrel $(\mathrm{mm})$ & 150.00 \\
Height of lower mandrel $(\mathrm{mm})$ & 245.00 \\
Radius of guide roll $(\mathrm{mm})$ & 300.00 \\
Cone angle of axial roll $\left({ }^{\circ}\right)$ & 35 \\
Initial outer radius of ring $(\mathrm{mm})$ & 900.0 \\
Initial inner radius of ring $(\mathrm{mm})$ & 600.0 \\
Initial height of ring $(\mathrm{mm})$ & 395.0 \\
Relative reduction ${ }^{*}$ of $\mathrm{UPR}(\mathrm{mm})$ & 1.31 \\
Rotational speed of driven roll $(\mathrm{mm} / \mathrm{s})$ & 1300 \\
Temperature of all rolls $\left({ }^{\circ} \mathrm{C}\right)$ & 200 \\
Temperature of ring blank $\left({ }^{\circ} \mathrm{C}\right)$ & 1200 \\
\hline Note ${ }^{*}$ Relative reduction $=\left(\left(R_{0}-r_{0}\right)-\left(R_{f}-r_{u f}\right)\right) /\left(R_{f}-r_{u f}\right)$ &
\end{tabular}

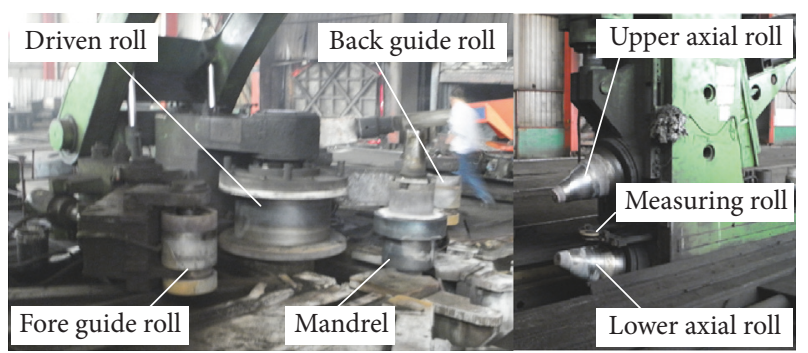

(a)

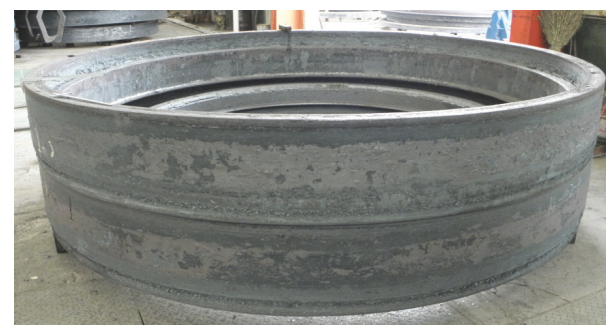

(b)

FIgURE 5: (a) Experimental ring rolling mill; (b) rolled inner L-shape rings.

is measured three times along the peripheral direction. The positions of the measured points are distributed uniformly along the peripheral direction. The average temperature values at different positions of the rolled ring were calculated.

Figure 7 shows the changing curves of ring's inner radius, ring's outer radius, and ring's height with rolling time. It can be seen that both ring's inner and outer radii increase stably in ILRRCDS, while the ring's height basically stays constant. At the end of ILRRCDS, the relative errors between the simulation values of ring's outer radius, inner radius of UPR, and inner radius of LPR and experimental ones

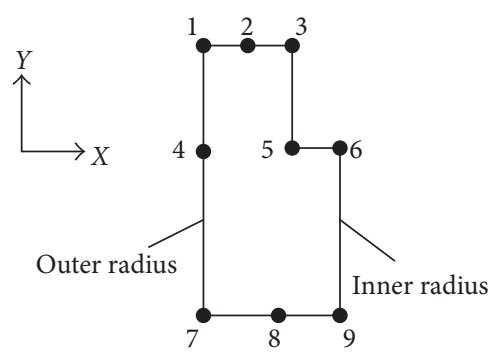

FIGURE 6: Temperature measuring point positions at the cross section of the rolled ring.

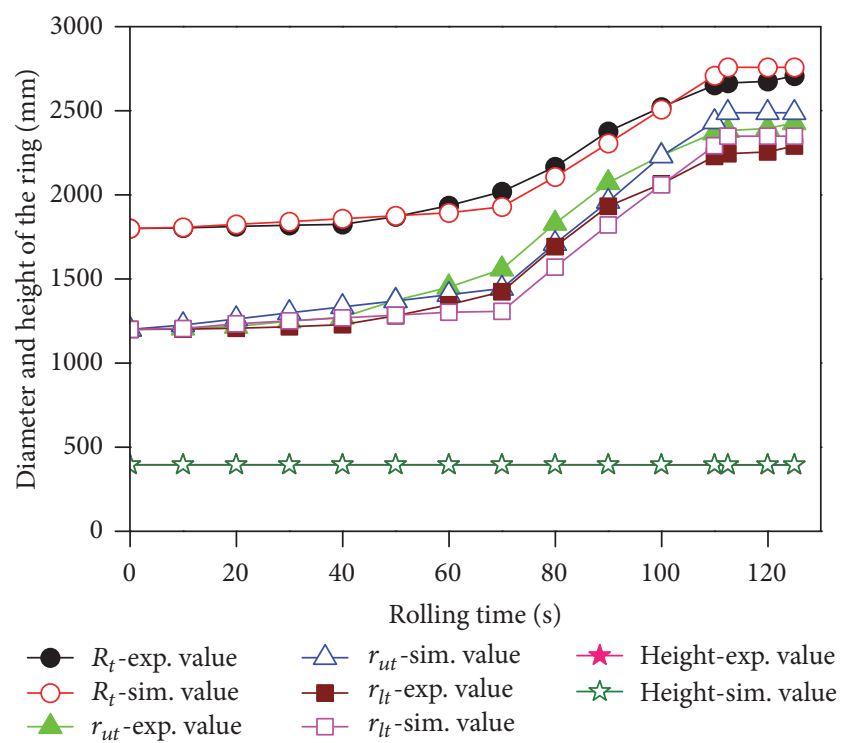

FIGURE 7: The changing curves of the ring's inner radius, ring's outer radius, and ring's height with rolling time.

are $-1.85 \%,-2.41 \%$, and $-2.47 \%$, respectively, where relative error is calculated by the following equation:

relative error

$$
\begin{aligned}
= & \left(\frac{(\text { experimental value }- \text { simulation value })}{\text { experimental value }}\right) \\
& \times 100 \% .
\end{aligned}
$$

Figure 8 shows the comparison between the experimental temperature values and simulative ones at different positions of rolled ring at $t=125 \mathrm{~s}$. It can be seen that the experimental temperature value at the outer surfaces of the ring at the end of the rolling process is a little lower than the simulation one. The maximum relative error which locates at position 7 is $-4.11 \%$. The reasons that caused the relative errors may be as follows: first, in realistic ILRRCDS, some water was ejected on the surface of the rolls in order to decrease the temperatures of rolls, and this water may increase the heat loss of the rolled ring. Second, it takes some time to move the rolled ring to an appropriate position at the end of ILRRCDS before measuring the rolled ring. This may increase the heat loss of the rolled ring because of the heat convection 


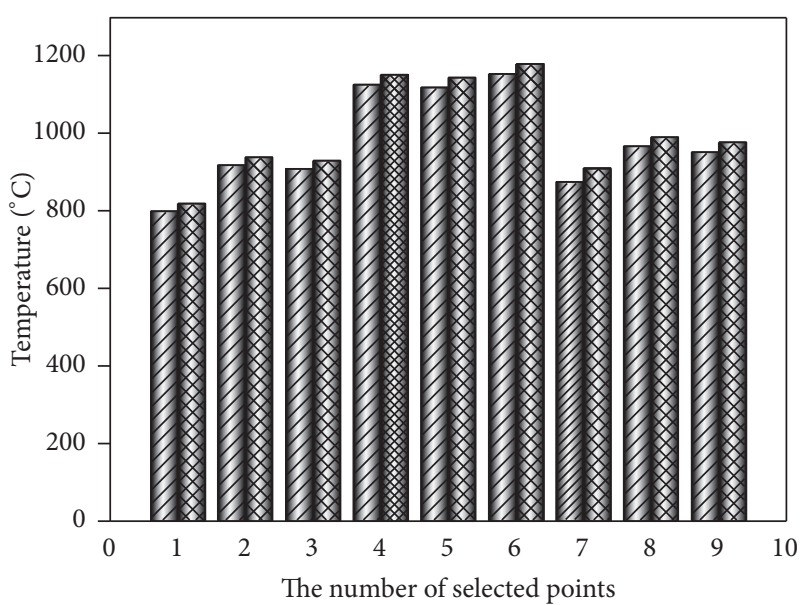

Exp. value
Sim. value

FIGURE 8: Comparison between the experimental temperature values and simulative ones at different positions of the rolled ring at $t=125 \mathrm{~s}$.

and heat radiation. Moreover, the error can also be caused by measurement, the selection of physical parameters, and the error of material constitutive model by Yang et al. [22]. Although there are some errors in experimental results, the relative errors are lower than 5\%. Thus, the FE model of ILRRCDS established in this paper is reliable.

\section{Results and Discussion}

This section studied the change laws of the equivalent plastic strain (PEEQ) and temperature distributions of rolled ring and rolling force and rolling moment with rolling time in ILRRCDS. The forming parameters in simulation are shown in Table 3.

5.1. The Change Laws of the PEEQ of Rolled Ring with Rolling Time. At the first forming stage, according to (21), when the mandrel feed rate is between $0.76 \mathrm{~mm} / \mathrm{s}$ and $4.46 \mathrm{~mm} / \mathrm{s}$, the plastic penetration condition and the biting-in condition can be simultaneously satisfied. In this simulative experiment, the mandrel feed rate is selected as $1 \mathrm{~mm} / \mathrm{s}$. The total feed amount is $70 \mathrm{~mm}$. Thus, the time, $\Delta T_{1}$, is calculated as $70.0 \mathrm{~s}$ at the first forming stage. According to (25), the reasonable value range of the growth rate of the ring's outer radius is between $2.53 \mathrm{~mm} / \mathrm{s}$ and $14.91 \mathrm{~mm} / \mathrm{s}$ at the second forming stage. The growth rate of the ring's outer radius is set as $10 \mathrm{~mm} / \mathrm{s}$. In this situation, the plastic penetration condition and the bitingin condition of the ring can be simultaneously satisfied. The time interval of the second forming stage, $\Delta T_{2}$, is calculated as $42.5 \mathrm{~s}$. At the roundness correction stage, the mandrel stopped moving towards the driven roll, and the time interval of the roundness correction stage, $\Delta T_{3}$, is $12.5 \mathrm{~s}$. Thus, the total rolling time is $125.0 \mathrm{~s}$.

Figure 9 shows the PEEQ distribution of rolled ring at different rolling times (PEEQ stands for the equivalent plastic strain). As is shown in Figure 9(a) at the first forming stage, the deformations of the ring mainly locate at both the inner and the outer radius surfaces of UPR stage. The value of the PEEQ at the inner radius surfaces of UPR is larger than the one at the outer radius surfaces of UPR. The common fishtail defects usually generated at the top end or bottom end surfaces of the ring are not found. In Figure 9(b), the LPR starts to be deformed at the end of the first forming stage. And the PEEQ at the inner radius surfaces of LPR is larger than the one at the outer radius surfaces of LPR. The outer radius value of UPR is larger than the one of LPR. Besides, the slight pulling and shrinking deformation of rolled ring is found. The reason may be that the metal of the UPR is rolled along the peripheral direction at the first forming stage of ILRRCDS, while the metal of the LPR is not rolled.

Figure 9(c) shows that, with the increase of rolling time, the wall thickness of UPR and LPR became thinner at the second forming stage, while the PEEQ at both the inner and the outer surfaces of the rolled ring became larger. Figure 9 (d) shows that the PEEQs at both the inner and the outer surfaces of the rolled ring are larger than the one at the middle of the ring at the end of the rolling process, which is similar to the deformation laws in radial-axial ring rolling process. Besides, the PEEQs at UPR are larger than the one at LPR. The minimum PEEQ locates at the middle part of LPR.

Generally, fishtail defects may occur at the radial plastic deformation zone in radial-axial ring rolling process, while this kind of defect does not occur in ILRRCDS. The main reasons can be explained from the following three aspects: first, the closed die structure at the top and bottom of the driven roll can effectively restrain the deformation of the ring along the axial direction. Second, the reasonable selected mandrel feed rate can maintain the stability of ILRRCDS. Third, the radial pulling and shrinking deformation is small as declared by Han [23]. The unwanted defects generated at the first forming stage can be improved at the second forming stage.

Figure 10(a) shows the initial ring blank and rolled ring. Since the PEEQ and temperature distributions of the inner Lshape ring are axis-symmetric about its center, a certain cross section of rolled L-shape ring "S-S" is selected to study the PEEQ and temperature distributions at $t=125 \mathrm{~s}$, as shown in Figures 10(b) and 10(c).

In Figure 10(b), ten points (from point A to point J) were selected to investigate the change laws of the PEEQ of the rolled ring with rolling time. Figure 11(a) shows the changing curves of the PEEQs at points A-J at the cross section "S-S" of rolled inner L-shape ring with rolling time. From Figures 9 and 11(a), the PEEQs at points A, C, and D basically increase linearly at first and finally keep unchanged at the roundness correction stage. At the end of the rolling process, the PEEQs at points $\mathrm{A}, \mathrm{C}$, and $\mathrm{D}$ are $2.8,3.5$, and 2.5 , respectively.

The PEEQs at points B, E, H, and I firstly increase slowly, then increase fast, and finally remain unchanged. At the end of the rolling process, the PEEQs at points $\mathrm{B}, \mathrm{E}, \mathrm{H}$, and $\mathrm{I}$ are $1.2,1.5,1.2$, and 1.8 , respectively. The reason is that the deformations mainly locate at the inner and outer radius surfaces of the rolled ring in the rolling process. This indicates that, with the increase of rolling time, the uniformity of the 

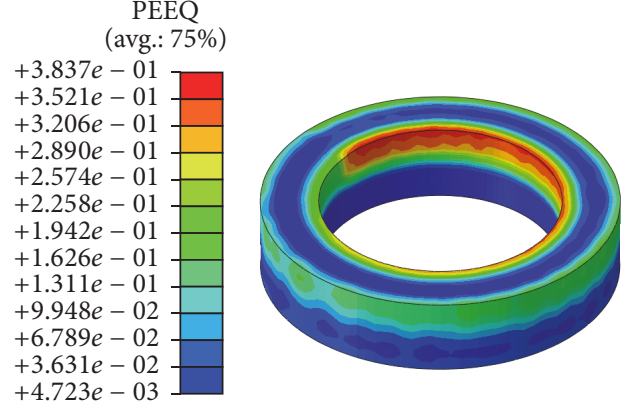

(a)
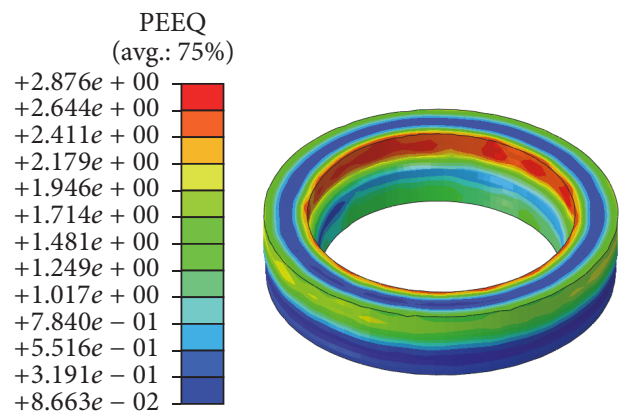

(b)

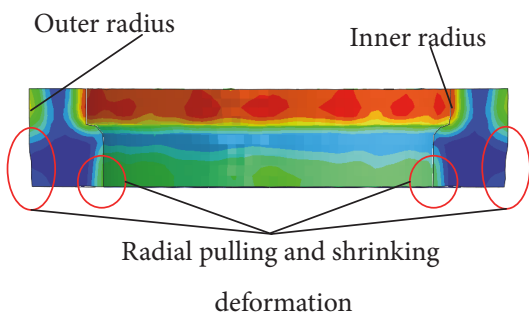

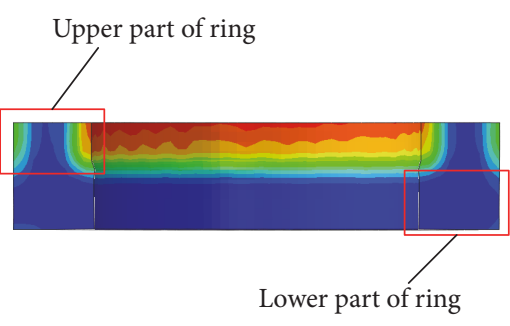

a) b)

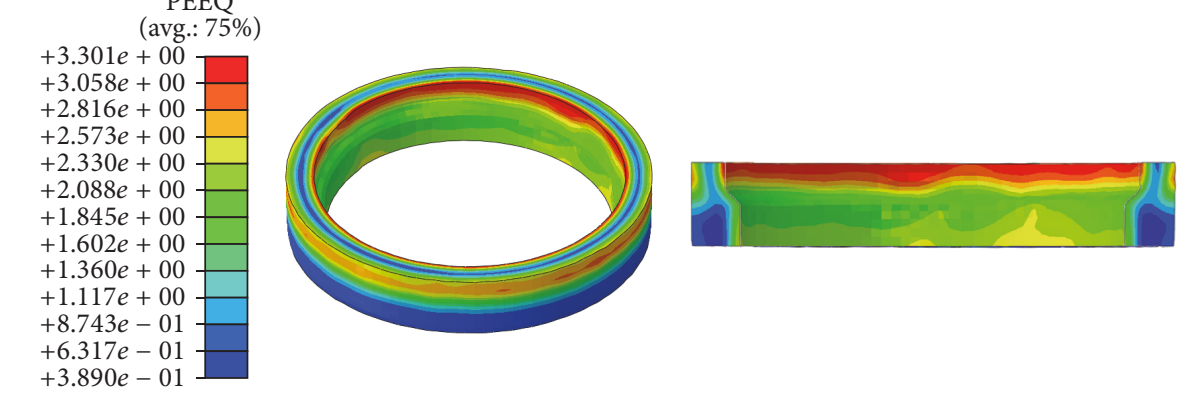

(c)
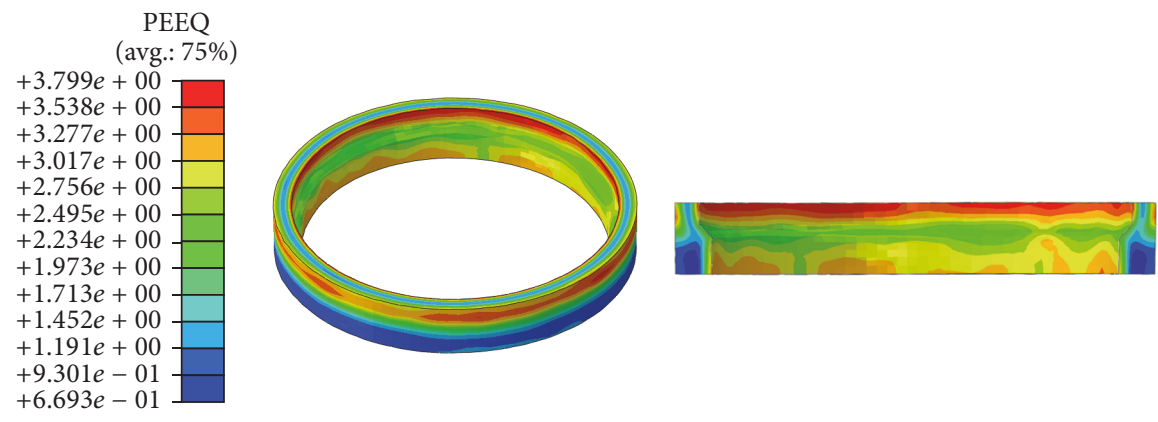

(d)

FIGURE 9: The equivalent plastic strain (PEEQ) distribution of the rolled ring at different rolling times: (a) $t=10 \mathrm{~s}$; (b) $t=70 \mathrm{~s}$; (c) $t=90 \mathrm{~s}$; (d) $t=125 \mathrm{~s}$.

PEEQ distribution of the rolled ring became worse. At the end of the rolling process, the larger values of the PEEQ at the inner radius surface of the rolled ring are 3.5 at point $\mathrm{C}$ and 3.1 at point J, respectively. The larger PEEQs at the outer radius surface of the rolled ring locate at point $A$ and point $H$. The simulative results indicate that the deformation at UPR is larger than the one at LPR, and the deformation at the inner radius surface of the rolled ring is larger than the one at the outer radius surface. Besides, according to ring rolling theory reported by Hua et al. [24], the feed amount per revolution of both the upper part of the mandrel, $\Delta h_{M 1}$, and the lower part of the mandrel, $\Delta h_{M 2}$, is larger than the feed amount of 


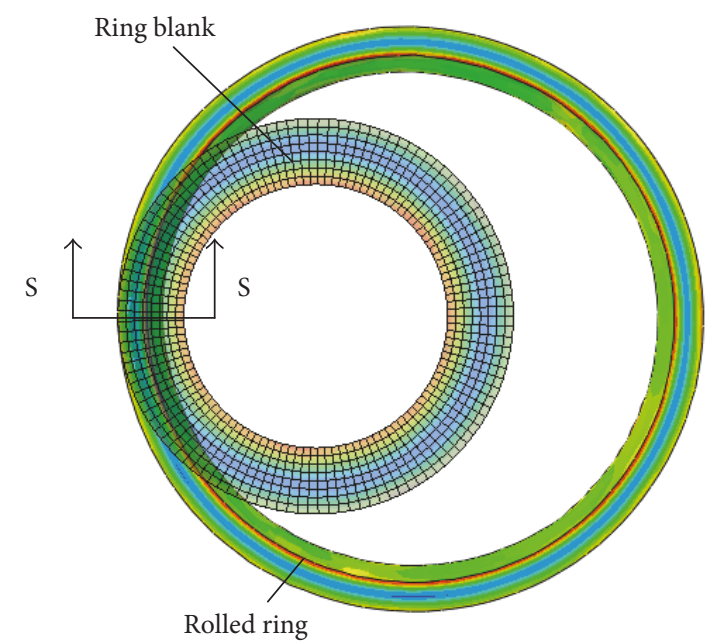

(a)
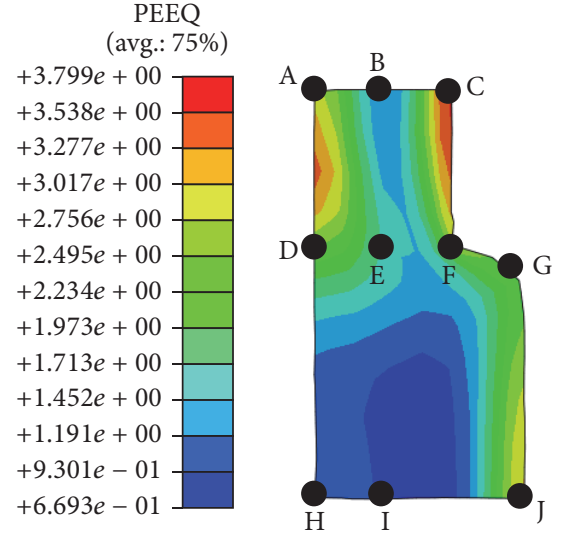

(b)

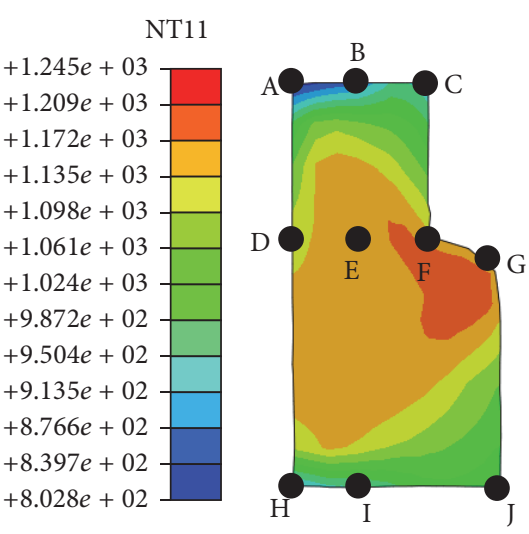

(c)

FIGURE 10: Simulation results at the end of the rolling process $(t=125 \mathrm{~s}$ ). (a) The initial ring blank and rolled ring; (b) the PEEQ distribution at the cross section "S-S" of the rolled inner L-shape ring; (c) the temperature distribution at the cross section "S-S" of the rolled inner L-shape ring (unit: ${ }^{\circ} \mathrm{C}$ ).

driven roll, $\Delta h_{D}$, in ILRRCDS. The simulative results are in good agreement with the calculated ones.

Moreover, it can be seen that the PEEQ at point F increases fast at the first forming stage and then increases slowly at the second forming stage. At the end of the rolling process, the PEEQ at point $\mathrm{F}$ is 2.7. This is because the metal at point $\mathrm{F}$ is rolled heavily and deformed seriously at the first forming stage while the metal at UPR moved downwards at the second forming stage, which supplied sufficient metal to deform along peripheral direction at point $\mathrm{F}$. This decreases the degree of deformation of the metal at point $\mathrm{F}$ along the peripheral direction to some extent.

It also can be seen that the metal at UPR deforms along the peripheral direction and $-Y$ direction from $0 \mathrm{~s}$ to about $60 \mathrm{~s}$, which leads to the pulling and shrinking deformation of the ring. In this situation, the metal at LPR is warped and deformed along the radial direction, and thus the time at which the lower part of the mandrel comes into contact with the metal at LPR (at about $t=60 \mathrm{~s}$ ) becomes earlier than the time predicted by the established mathematical model (at $t=$ $70 \mathrm{~s})$.
Figures $11(\mathrm{~b})$ and $11(\mathrm{c})$ are at $t=125 \mathrm{~s}$, the changing curves of the PEEQ at the cross section "S-S" of rolled ring along the radial direction and along the axial direction, respectively. In Figures 10(b) and 11(b), the PEEQs of both line ABC and line $\mathrm{HIJ}$ along the radial direction from outer radius to inner radius of the ring decrease at first and then increase. The PEEQ of line DEFG decreases at first, then increases, and finally slightly decreases. This is because, according to plain ring rolling theory, the metals at both the inner and the outer radius surfaces of the ring are easier penetrated than the one at the middle part of the ring in the rolling process.

It also can be seen that the PEEQs at the UPR are larger than the ones at both the inner and the outer radius surfaces of LPR. The main reason is that the total deformation amount of UPR is larger than the one of LPR. The PEEQ at point $\mathrm{F}$ is larger than the one at point $\mathrm{G}$. It can be deduced that the grain sizes at points A, C, D, F, G, and J are fine due to the larger PEEQ at these points, but these positions are the potential areas where cracks may occur due to their severe deformation. 


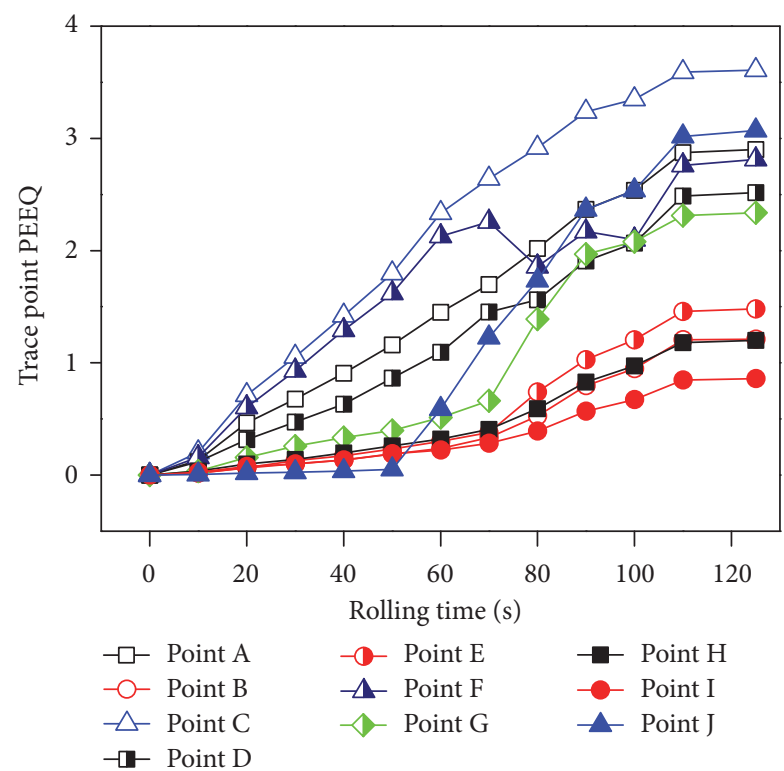

(a)

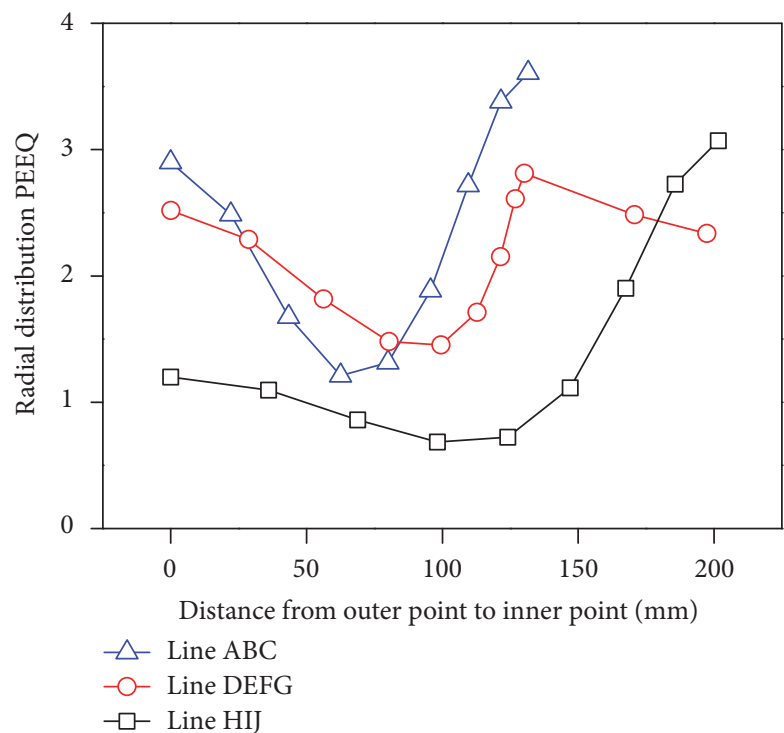

(b)

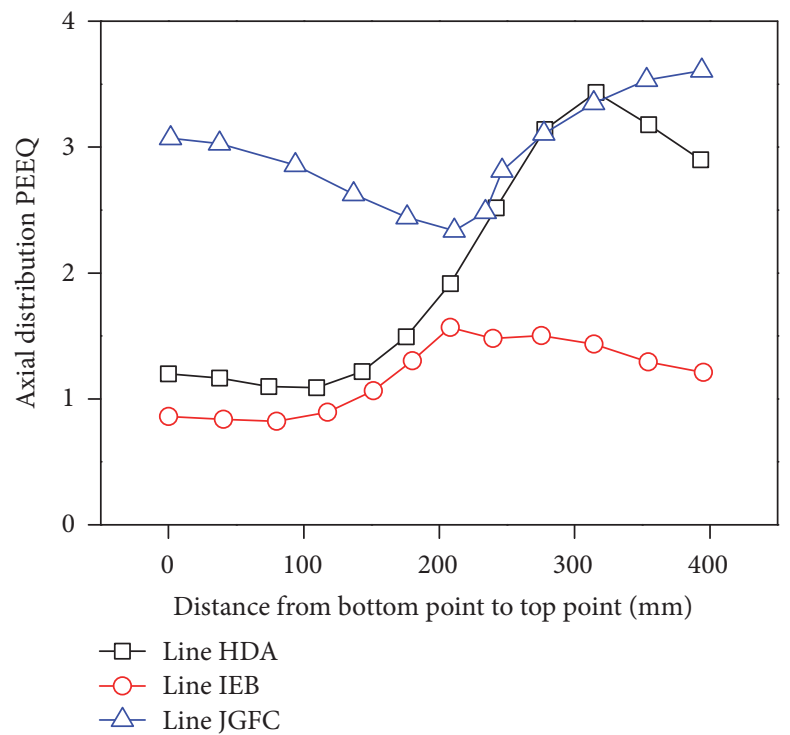

(c)

FIgURE 11: The changing curves of the PEEQ of the rolled ring. (a) The changing curves of the PEEQ of points A-J with rolling time; (b) the changing curves of the PEEQ at the cross section "S-S" of the rolled ring along the radial direction $(t=125 \mathrm{~s})$; (c) the changing curves of the PEEQ at the cross section "S-S" of the rolled ring along the axial direction ( $t=125 \mathrm{~s})$.

From Figures 10(b) and 11(c), the PEEQ of line JGFC along the axial direction from the bottom of the ring to the top of the ring gradually decreases and then increases. The maximum PEEQ located at the zone near point $\mathrm{C}$. The minimum PEEQ located at the zone near point G. The PEEQ of line IEB gradually increases and then slightly decreases. The maximum PEEQ located at the zone near point E. The minimum PEEQ located at the zone near point I. The PEEQ of line HDA gradually increases and then slightly decreases. The maximum PEEQ located at the zone between point $A$ and point $\mathrm{D}$. The minimum PEEQ located at the zone between point $\mathrm{D}$ and point $\mathrm{H}$.
The reason can be explained from the following two aspects: on the one hand, the temperature at point $\mathrm{A}$ is lower due to its large heat loss, and thus the flow property of the metal at point $\mathrm{A}$ is worse than the one at the zone between point $\mathrm{A}$ and point $\mathrm{D}$. On the other hand, the metal deformation accumulation at point $\mathrm{D}$ is smaller.

\subsection{The Change Laws of the Temperature Distribution of Rolled} Ring with Rolling Time. Figure 12 shows the temperature distributions of deforming ring at different rolling times. In Figure 12(a), the temperature values at the top end surface, the bottom end surface, the outer radius surface, and the inner 


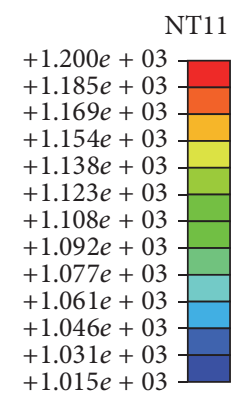

NT11

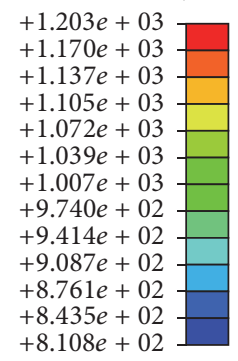

NT11

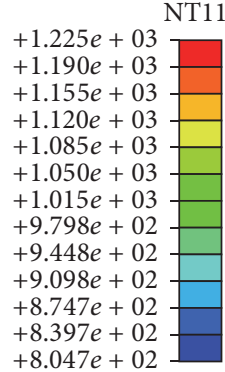

NT11

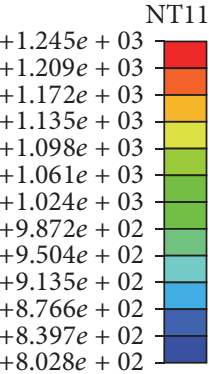

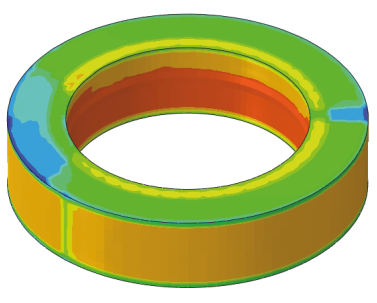

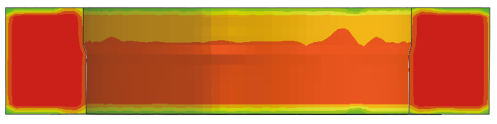

(a)
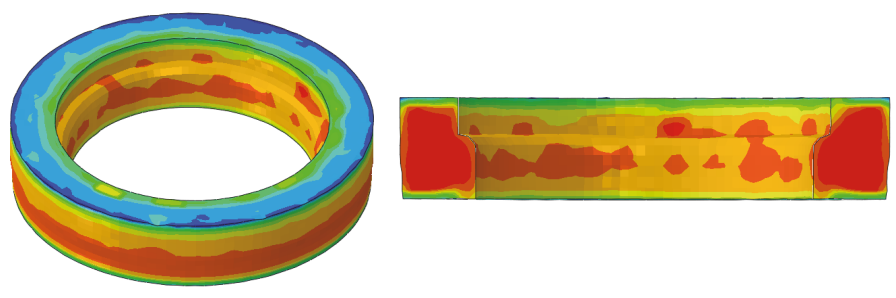

(b)
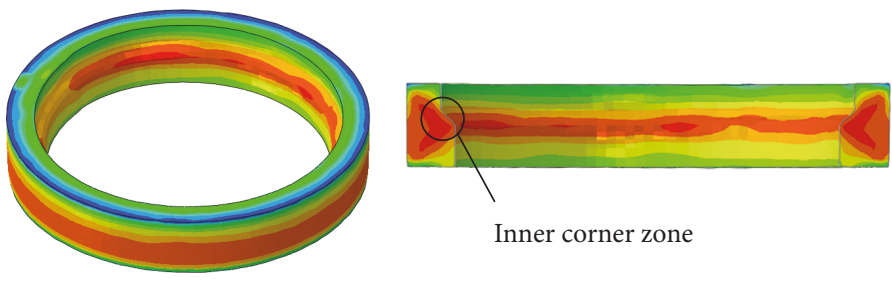

(c)
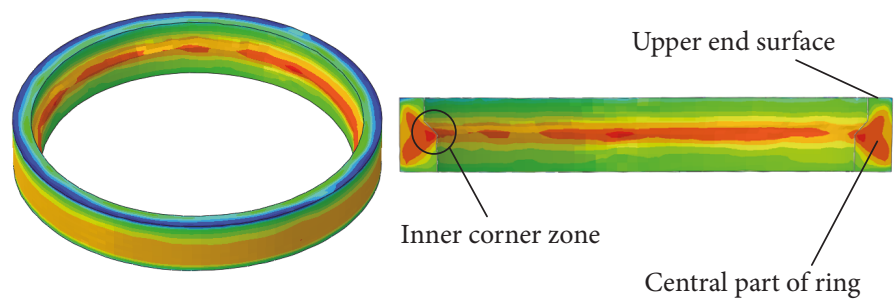

(d)

Figure 12: The temperature distributions of deforming ring at different rolling times: (a) $t=10 \mathrm{~s}$; (b) $t=70 \mathrm{~s}$; (c) $t=90 \mathrm{~s}$; (d) $t=125 \mathrm{~s}$ (unit: $\left.{ }^{\circ} \mathrm{C}\right)$.

radius surface of UPR are larger due to the large heat loss caused by the heat conduction between the ring and rolls, such as the driven roll, axial rolls, and the upper part of the mandrel. The temperature value at the inner radius surface of LPR is small because the heat loss at the inner radius surface of LPR is small. Figure 12(b) shows the temperature distribution of deforming ring at the end of the first forming stage. In Figure 12(b), the temperature at the surfaces of the ring is smaller than the one at the center of the ring. The temperatures at the top end surface and the bottom end surface of the ring are smaller than those at other positions. The reason is that, on the one hand, the heat conduction between the ring and the rolls, such as the driven roll, axial rolls, and the upper part of the mandrel, leads to more heat loss. On the other hand, the heat convection and heat radiation between the ring's surfaces and the air also cause heat loss. Figure 12(c) shows the temperature distribution of deforming ring at $t=90 \mathrm{~s}$. The high temperature zone 
gradually moves form the center part of the ring to the "inner corner zone" of the L-shape ring. Figure 12(d) shows the temperature distribution of deforming ring at $t=125 \mathrm{~s}$. The maximum temperature zone is near the "inner corner zone" of the L-shape ring.

Generally, the temperature distribution of the rolled ring is axis-symmetric about its center in the rectangular radialaxial ring rolling process. The temperatures at both the outer and the inner radius surfaces of the ring are lower than the one at the center part of the ring. However, the high temperature zone moves to the "inner corner zone" in the inner L-shape ring rolling process. The main reason is as follows: first, the deformation of the ring is asymmetric. The deformation of UPR is larger than the deformation of LPR. And thus, the uniformity of the distribution of heat generated by metal deformation becomes worse. Second, the contact area between the LPR and the lower part of the mandrel is large. The contact time between the LPR and the lower part of the mandrel is too long. In this situation, the heat loss of UPR is larger than the one of LPR. Third, the specific surface area at the "inner corner zone" of the ring is small, which is the disadvantage of heat convection and heat radiation. On the contrary, the heat loss at other zones caused by heat convection and heat radiation is large due to their large specific surface area.

In Figure 10(b), ten points (from point A to point J) were selected to investigate the change laws of the temperature of the rolled ring with rolling time. Figure 13(a) shows the changing curves of the temperature values at points A-J with rolling time. The temperatures at points $\mathrm{D}, \mathrm{E}, \mathrm{F}$, and $\mathrm{G}$ positions are basically above $1140^{\circ} \mathrm{C}$ in the whole ILRRCDS. However, the temperatures at other positions gradually decrease with rolling time. The temperatures at points $\mathrm{A}$ to $\mathrm{J}$ positions are above $830^{\circ} \mathrm{C}$, at the end of the rolling process. The temperature at point $G$ decreases at first, then increases, and finally keeps unchanged. Its final temperature is $1180^{\circ} \mathrm{C}$. This may be because the heat loss at point $\mathrm{G}$ is too large at the first forming stage and the heat generated by metal deformation at point $G$ is larger than the heat loss at point $G$ at the second forming stage. Thus, the temperature at point $\mathrm{G}$ slightly increases. The temperatures at points $\mathrm{B}, \mathrm{H}$, and I positions gradually decrease at the first forming stage, slightly increase at the second forming stage, and finally decrease. The final temperatures at points $\mathrm{B}, \mathrm{H}$, and $\mathrm{I}$ positions are $880^{\circ} \mathrm{C}, 920^{\circ} \mathrm{C}$, and $960^{\circ} \mathrm{C}$, respectively. The reason may be that the generated heat at these positions from $70 \mathrm{~s}$ to $90 \mathrm{~s}$ is larger than the heat loss at these positions, but the generated heat at these positions from $90 \mathrm{~s}$ to $125 \mathrm{~s}$ is smaller than the heat loss at these positions. Moreover, the temperatures at points $\mathrm{A}, \mathrm{B}, \mathrm{C}$, and $\mathrm{H}$ positions are $830^{\circ} \mathrm{C}$, $880^{\circ} \mathrm{C}, 925^{\circ} \mathrm{C}$, and $920^{\circ} \mathrm{C}$, respectively. The temperatures at points $\mathrm{A}, \mathrm{B}, \mathrm{C}$, and $\mathrm{H}$ positions are smaller than the ones at other positions due to the larger heat loss and smaller generated heat at these positions.

Figures 13(b) and 13(c) show, at $t=125 \mathrm{~s}$, the changing curves of the temperature of the rolled ring along the radial direction and the axial direction, respectively. From Figures 10(c) and 13(b), the temperatures at line ABC and line $\mathrm{HIJ}$ along the radial direction from the ring's inner radius to the ring's outer radius both increase at first and then slightly decrease. The temperatures at line DEFG increase at first, then decrease, and finally increase slightly. Besides, the temperatures at the middle of the ring, at the bottom end surface, and at the top end surface decrease systematically. In Figures 10(c) and 13(c), the temperatures at line JGFC and line HDA along the axial direction from the bottom end surface of the ring to the top end surface of the ring both gradually increase at first and then decrease. The temperatures at line IEB increase firstly, then keep unchanged, and finally decrease.

From Figures 10(c), 13(b), and 13(c), it can be seen that the temperature at the center part of the ring is larger than the ones at the surfaces of the ring. The difference between the temperatures at the surfaces of the ring and the one at the corner zone is large. The temperatures at points $\mathrm{A}, \mathrm{C}, \mathrm{H}$, and $J$ positions are lower. This may be explained from the following two aspects: on the one hand, the heat loss at these points is larger due to the larger heat conduction between the ring and the rolls. On the other hand, the specific surface areas at points $\mathrm{A}, \mathrm{C}, \mathrm{H}$, and $\mathrm{J}$ positions are large, and thus the heat loss is large. The temperatures at points $\mathrm{A}, \mathrm{C}$, and $\mathrm{H}$ positions are lower than the temperature at point J position. The reason may be that, first, the heat losses at points $\mathrm{A}, \mathrm{C}$, and $\mathrm{H}$ positions are larger than the heat loss at point J position because there is heat conduction between the UPR with the driven roll and the upper part of the mandrel. Second, the heat generated by metal deformation at point $\mathrm{H}$ position is lower than the one generated by metal deformation at point $J$ position because the metal at point $J$ position deforms from about $60 \mathrm{~s}$ to $112 \mathrm{~s}$ while the metal at point $\mathrm{H}$ position deforms from about $70 \mathrm{~s}$ to $112 \mathrm{~s}$. Third, according to the ring rolling theory, the feed amount per revolution of mandrel is larger than the one of driven roll, which leads to larger metal deformation amount and larger generated heat at point J position.

In Figure 13(b), the change law of rolled ring's temperature along line DEF is similar to the one along line ABC. The main reason is that the total feed amount of the ring at line DEF is equal to the one at line $\mathrm{ABC}$. The change law of rolled ring's temperature along line FG is slightly different from that along line IJ. The main reason is that the total feed amount of the ring at line FG is equal to the one at line IJ, but the heat loss at point $G$ position is smaller than the one at point J position. It can be deduced that the metal at line FG may be destroyed because the metal at the position suffers residual hot tensile stress.

\subsection{The Change Laws of Rolling Force and Rolling Moment} with Rolling Time. Figure 14 shows the changing curve of rolling force with rolling time. The rolling forces of both the driven roll and the mandrel gradually increase at the first forming stage. The rolling forces of both the driven roll and the mandrel seriously increase at first and then keep unchanged at the second forming stage. The rolling forces of both the driven roll and the mandrel gradually decrease at the roundness correction stage. In the whole ILRRCDS, the rolling force of the mandrel is maximum; the rolling force of 


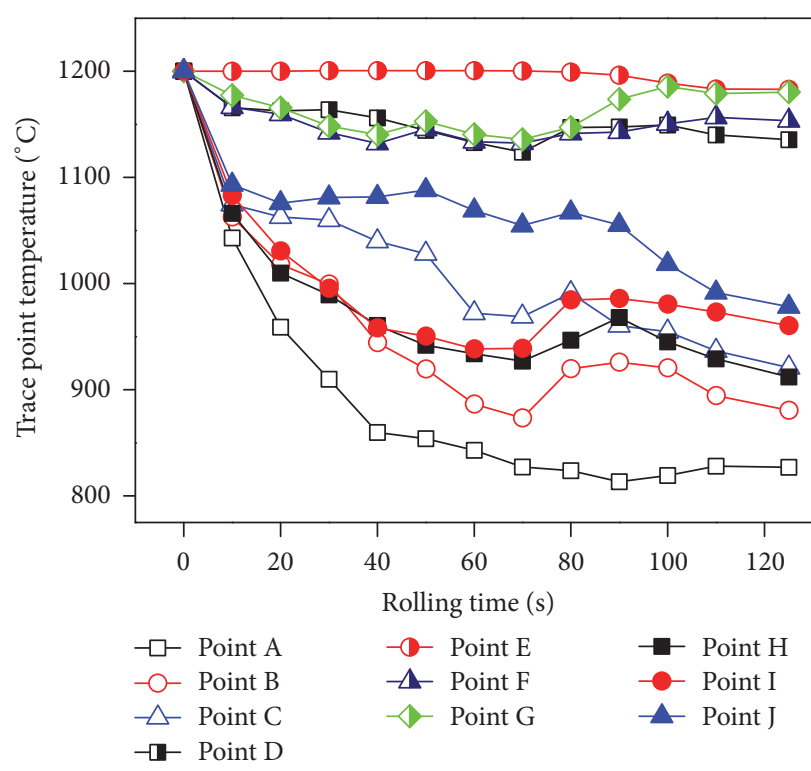

(a)

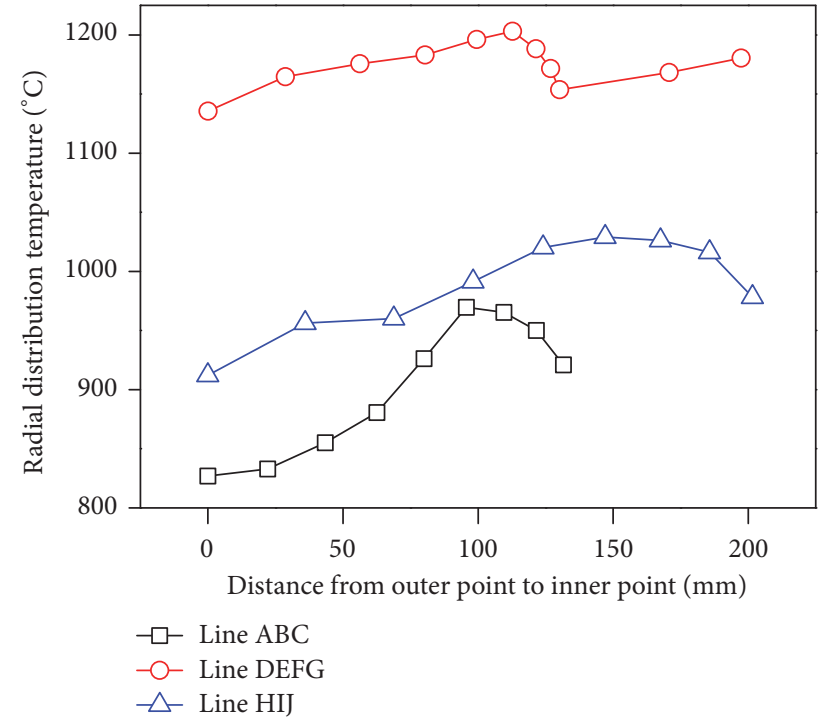

(b)

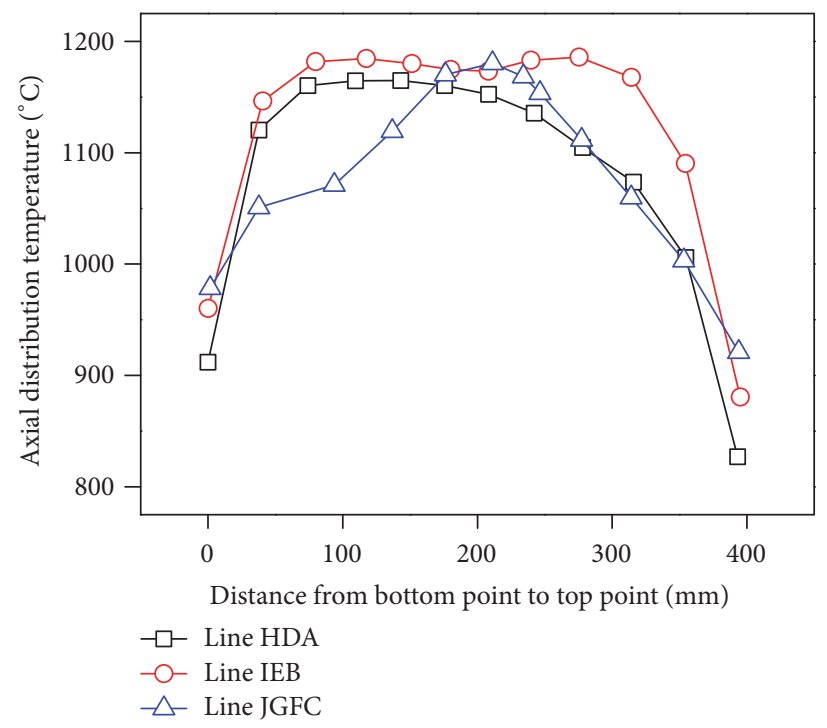

(c)

FIGURE 13: The changing of the temperature of the rolled ring. (a) The changing curves of the temperature of points A-J with rolling time; (b) the changing curves of the temperature along the radial direction $(t=125 \mathrm{~s})$; (c) the changing curves of the temperature along the axial direction $(t=125 \mathrm{~s})$.

the driven roll takes the second place; and the rolling force of the axial rolls is minimum.

When the rolling time is around $20 \mathrm{~s}$, the rolling force of the upper axial roll exists but the rolling force of the lower axial roll is zero. This indicates that a warped deformation along the $Y$-axis may occur in the axial plastic deformation zone. When the rolling time is around $50 \mathrm{~s}, 70 \mathrm{~s}, 117.5 \mathrm{~s}$, and $125 \mathrm{~s}$, the rolling force of the lower axial roll exists but the rolling force of the upper axial roll is zero. This indicates that a warped deformation along the $-Y$-axis direction may occur in the axial plastic deformation zone. When the rolling time is around $60 \mathrm{~s}$ and $100 \mathrm{~s}$, the rolling forces of both the upper axial roll and the lower axial roll exist. This may be because the pulling and shrinking deformation along the radial direction in the rolling process leads to warped deformations along the $Y$-axis at the inner radius surface of the ring and along the $-Y$-axis at the outer radius surface of the ring.

Figure 15 shows the changing curve of rolling moment with rolling time. The change laws of rolling moment with rolling time are similar to the change laws of rolling force with rolling time. In the whole ILRRCDS, the rolling moment of the mandrel is maximum; the rolling moment of the driven roll takes the second place; and the rolling moment of axial rolls is minimum. The metal deformation of the ring along the axial direction is seriously restricted by the closed die structure of the top and bottom of the driven roll. 


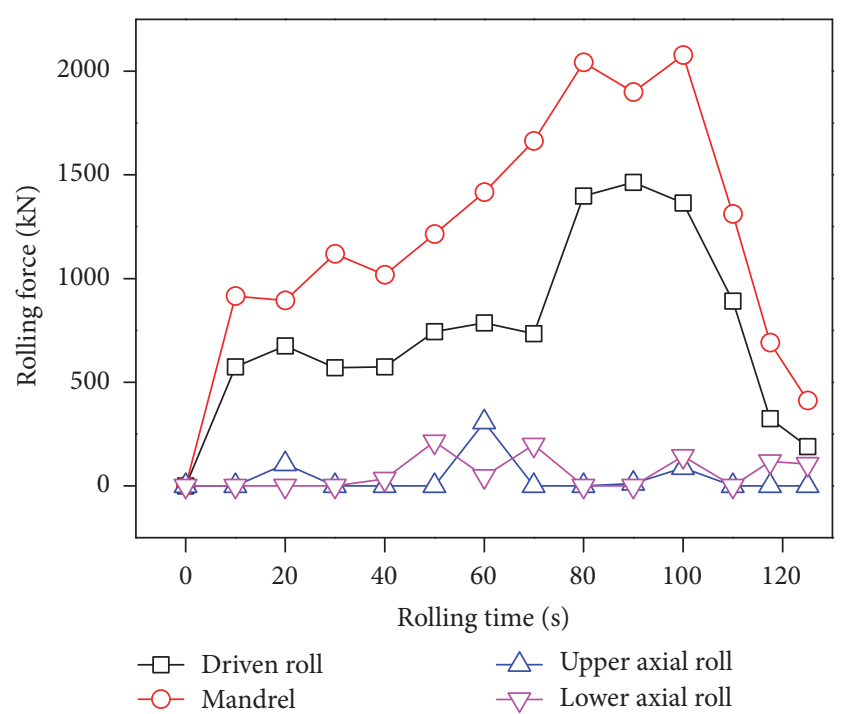

FIGURE 14: The changing curve of rolling force with rolling time.

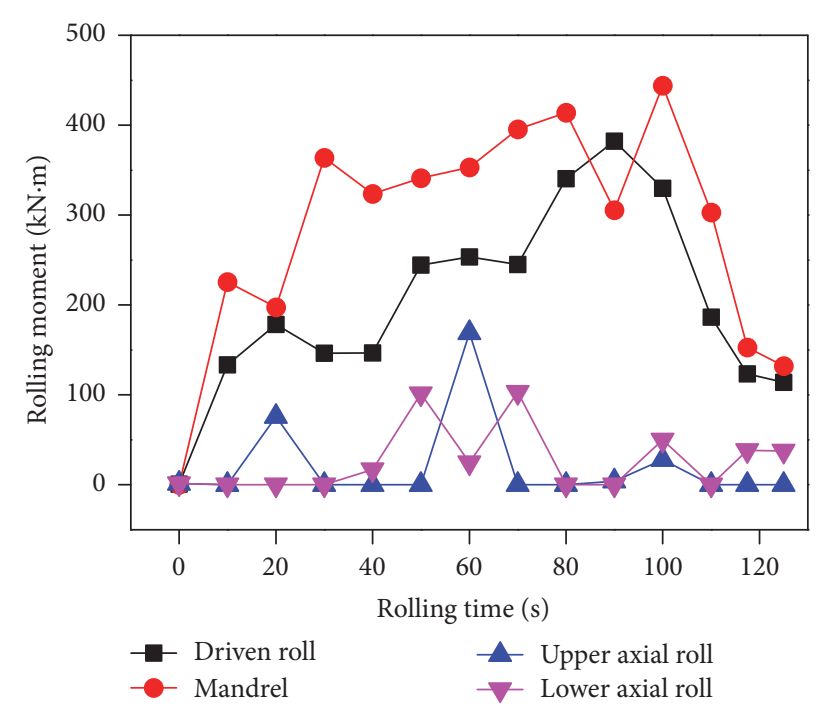

FIGURE 15: The changing curve of rolling moment with rolling time.

\section{Conclusions}

This paper established the mathematical model for ILRRCDS, deduced the plastic penetration and biting-in conditions of the ring in ILRRCDS, proposed a feeding strategy that can realize a constant growth for the ring's outer radius, and determined the reasonable value ranges of the feed rate of the mandrel. Finally, both the PEEQ and the temperature distributions of rolled ring and the change laws of the rolling force and rolling moment with rolling time were obtained. The main conclusions are drawn as follows:

(1) The plastic penetration and biting-in conditions of the UPR should satisfy $\Delta h_{\min , u 1} \leq \Delta h_{u 1} \leq \Delta h_{\max , u 1}$ at the first forming stage; the plastic penetration and biting-in conditions of both the UPR and the LPR at the second forming stage should satisfy the following formulae:

$$
\begin{aligned}
& \Delta h_{2, \min }=\max \left\{\Delta h_{\min , u 2}, \Delta h_{\min , l 2}\right\}, \\
& \Delta h_{2, \max }=\min \left\{\Delta h_{\max , u 2}, \Delta h_{\max , l 2}\right\}, \\
& \Delta h_{2, \min } \leq \Delta h_{2} \leq \Delta h_{2, \text { max }} .
\end{aligned}
$$

The reasonable value ranges of $v_{R 2}$ should satisfy the following formula:

$$
\begin{aligned}
& f_{2}(t) \\
& \cdot\left[\frac{H\left[V_{0}+\pi H_{M 1}\left(b_{l t}-R_{M 1}+R_{M 2}\right)^{2}+\pi H_{M 2} b_{l t}^{2}\right]}{2 \pi\left[H_{M 1}\left(b_{l t}-R_{M 1}+R_{M 2}\right)+H_{M 2} b_{l t}\right]^{2}}\right. \\
& -1] \leq v_{R 2} \leq g_{2}(t) \\
& \cdot\left[\frac{H\left[V_{0}+\pi H_{M 1}\left(b_{l t}-R_{M 1}+R_{M 2}\right)^{2}+\pi H_{M 2} b_{l t}^{2}\right]}{2 \pi\left[H_{M 1}\left(b_{l t}-R_{M 1}+R_{M 2}\right)+H_{M 2} b_{l t}\right]^{2}}\right. \\
& \quad-1] .
\end{aligned}
$$

(2) The proposed feeding strategy can realize a stable ILRRCDS. And simulative results indicate that the pulling and shrinking deformations occurred at LPR at the first forming stage in ILRRCDS while these deformations were improved at the second forming stage. At the end of ILRRCDS, the PEEQ at the inner radius surface of the rolled ring is maximum; the PEEQ at the outer radius surface of the rolled ring takes the second place; and the PEEQ at the middle part of the rolled ring is minimum.

(3) The differences between the temperature at the center part of the rolled ring and those at the surface of the ring are great. The temperature at the center part of the rolled ring at the first forming stage is higher than the one at the surface of the rolled ring. The higher temperature zone gradually moves from the center part of the rolled ring to the "inner corner zone" of the rolled ring. At the end of the rolling process, the temperatures at the middle of the rolled ring, at the bottom end surface of the rolled ring, and at the top end surface of the rolled ring decreased in turn.

(4) The rolling forces and rolling moments of both the driven roll and the mandrel gradually increase at the first forming stage. The rolling forces and rolling moments of both the driven roll and the mandrel seriously increase at first and then keep unchanged at the second forming stage. The rolling forces and rolling moments of both the driven roll and the mandrel gradually decrease at the roundness correction stage. In the whole ILRRCDS, the rolling force and 
rolling moment of the mandrel are maximum; the rolling force and rolling moment of the driven roll take the second place; and the rolling force and rolling moment of the axial rolls are minimum.

\section{Conflicts of Interest}

The authors declare that there are no conflicts of interest regarding the publication of this paper.

\section{References}

[1] J. M. Allwood, A. E. Tekkaya, and T. F. Stanistreet, "The development of ring rolling technology," Steel Research International, vol. 76, no. 2-3, pp. 111-120, 2005.

[2] J. M. Allwood, R. Kopp, D. Michels et al., "The technical and commercial potential of an incremental ring rolling process," CIRP Annals - Manufacturing Technology, vol. 54, no. 1, pp. 233236, 2005.

[3] L. Li, H. Yang, L. Go, and Z. Sun, "Research on interactive influences of parameters on T-shaped cold ring rolling by $3 \mathrm{~d}$ FE numerical simulation," Journal of Mechanical Science and Technology, vol. 21, no. 10, pp. 1541-1547, 2007.

[4] K. H. Kim, H. G. Suk, and M. Y. Huh, "Development of the profile ring rolling process for large slewing rings of alloy steels," Journal of Materials Processing Technology, vol. 187-188, pp. 730733, 2007.

[5] L. Hua, D.-S. Qian, and L.-B. Pan, "Deformation behaviors and conditions in L-section profile cold ring rolling," Journal of Materials Processing Technology, vol. 209, no. 11, pp. 5087-5096, 2009.

[6] H. Yang, L. Li, M. Wang, and L. Guo, "Research on the expanding deformation of ring radius in cold profiled ring rolling process," Science China Technological Sciences, vol. 53, no. 3, pp. 813-821, 2010.

[7] Y. S. Lee, M. W. Lee, S. S. Park, I. Lee, and Y. H. Moon, "Process design by FEM simulation for shape ring rolling of large-sized ring," in Proceedings of the 10th International Conference on Numerical Methods in Industrial Forming Processes Dedicated to Professor O. C. Zienkiewicz (1921-2009), NUMIFORM 2010, pp. 964-971, kor, June 2010.

[8] A. Kluge, Y.-H. Lee, H. Wiegels, and R. Kopp, "Control of strain and temperature distribution in the ring rolling process," Journal of Materials Processing Tech., vol. 45, no. 1-4, pp. 137-141, 1994.

[9] L. Guo and H. Yang, "Towards a steady forming condition for radial-axial ring rolling," International Journal of Mechanical Sciences, vol. 53, no. 4, pp. 286-299, 2011.

[10] N. Kim, H. Kim, and K. Jin, "Optimal design to reduce the maximum load in ring rolling process," International Journal of Precision Engineering and Manufacturing, vol. 13, no. 10, pp. 1821-1828, 2012.

[11] W. Xu, X. Yang, Z. Jiang, and Q. Wang, "Feeding strategy design for steel $42 \mathrm{CrMo}$ in radial-axial ring rolling using processing map," International Journal of Material Forming, pp. 1-8, 2013.

[12] L. Tian, Y. Luo, H.-J. Mao, and L. Hua, "A hybrid of theory and numerical simulation research for virtual rolling of doublegroove ball rings," International Journal of Advanced Manufacturing Technology, vol. 69, no. 1-4, pp. 1-13, 2013.

[13] L. Li, X. Li, J. Liu, and Z. He, "Modeling and simulation of cold rolling process for double groove ball-section ring,"
International Journal of Advanced Manufacturing Technology, vol. 69, no. 5-8, pp. 1717-1729, 2013.

[14] L. Giorleo, C. Giardini, and E. Ceretti, "Validation of hot ring rolling industrial process 3D simulation," International Journal of Material Forming, vol. 6, no. 1, pp. 145-152, 2013.

[15] J. Li, X. Zhang, and J. Liu, "Hot deformation behavior and dynamic Recrystallization diagram for low-alloy Q345E steel at high temperature," J. Forging \& Stamping Technology, vol. 38, pp. 148-151, 2013.

[16] L. Hua and Z. Z. Zhao, "The extremum parameters in ring rolling," Journal of Materials Processing Technology, vol. 69, no. 1-3, pp. 273-276, 1997.

[17] L. B. Pan, On deformation laws and CAPP system for radial-axial ring rolling [Doctoral, thesis], Doctoral Dissertation for Wuhan University of Technology, Wuhan, 2007.

[18] K.-H. Lee and B.-M. Kim, "Advanced feasible forming condition for reducing ring spreads in radial-axial ring rolling," International Journal of Mechanical Sciences, vol. 76, pp. 21-32, 2013.

[19] “ABAQUS 6.14 User's Manual, 2014”.

[20] G. Zhou, L. Hua, J. Lan, and D. S. Qian, "FE analysis of coupled thermo-mechanical behaviors in radial-axial rolling of alloy steel large ring," Computational Materials Science, vol. 50, no. 1, pp. 65-76, 2010.

[21] K. Shiro, O. Soo-ik, and A. Taylan, Metal Forming and the Finite Element Method, Oxiford University Press, New York, USA, 1989.

[22] H. Yang, M. Wang, L. G. Guo, and Z. C. Sun, “3D coupled thermo-mechanical FE modeling of blank size effects on the uniformity of strain and temperature distributions during hot rolling of titanium alloy large rings," Computational Materials Science, vol. 44, no. 2, pp. 611-621, 2008.

[23] X. H. Han, The investigation of rolling technology and equipment in conical ring rolling with inner steps, Masters' Dissertation for Wuhan University of Technology, Wuhan, China, 2006.

[24] L. Hua, X. G. Huang, and C. D. Zhu, Theory and Technology of Ring Rolling, Mechanical Industry Press, Beijing, China, 2001. 


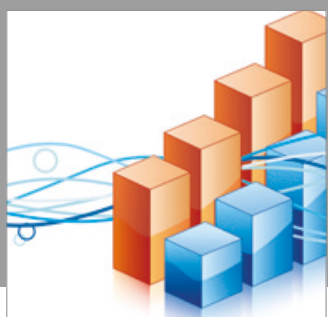

Advances in

Operations Research

vatersals

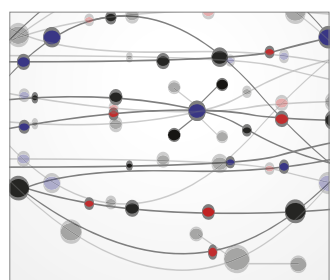

\section{The Scientific} World Journal
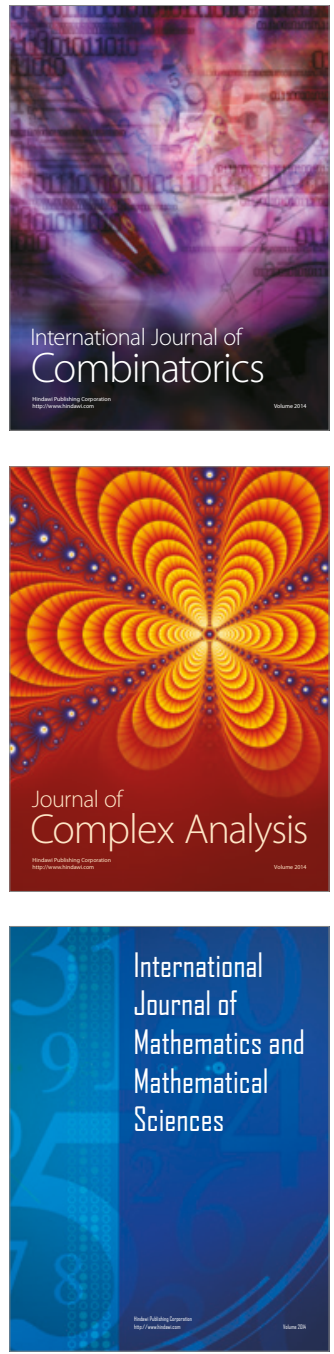
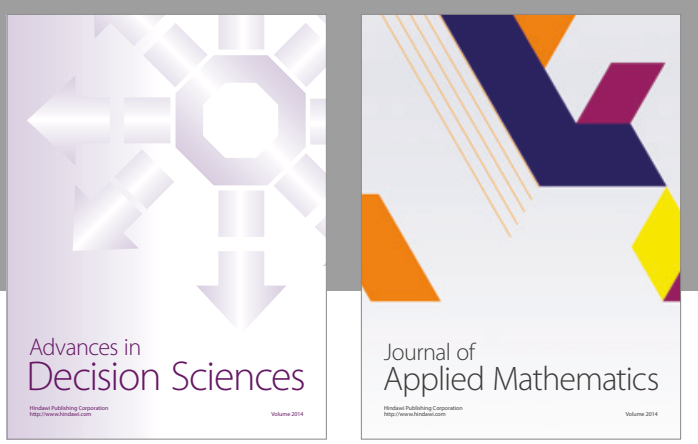

Algebra

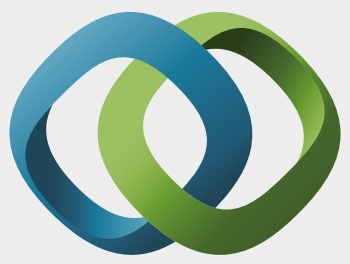

\section{Hindawi}

Submit your manuscripts at

https://www.hindawi.com
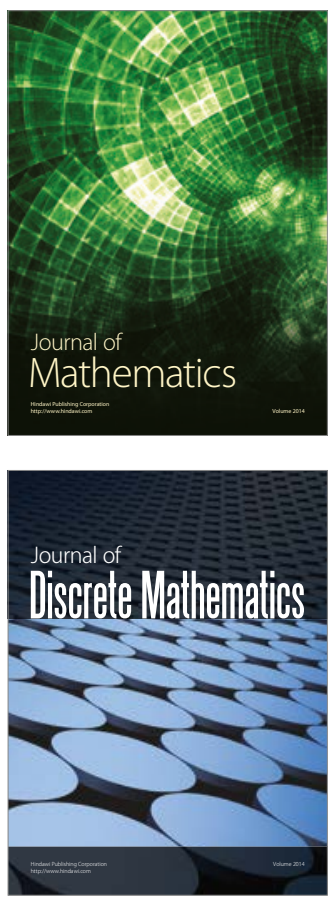

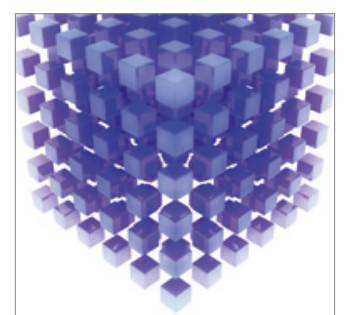

Mathematical Problems in Engineering
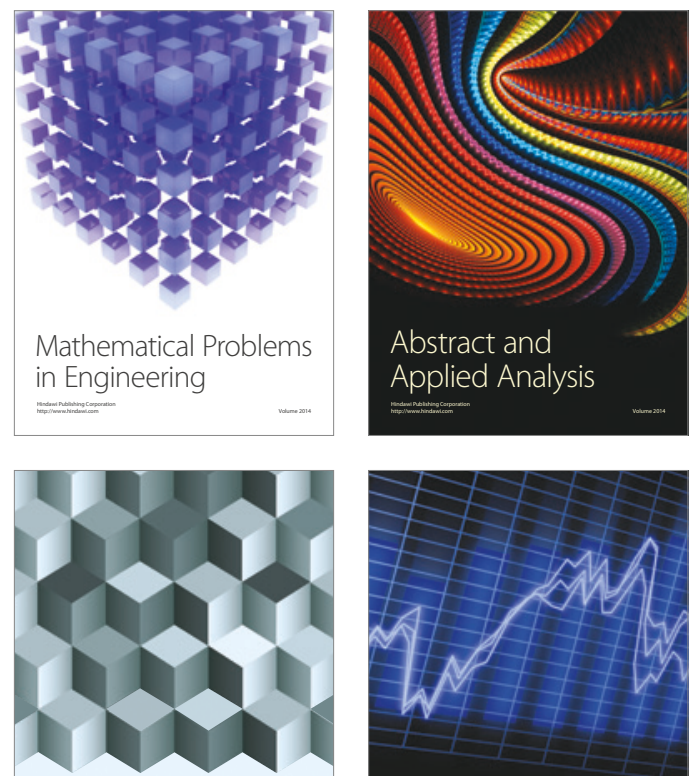

Journal of

Function Spaces

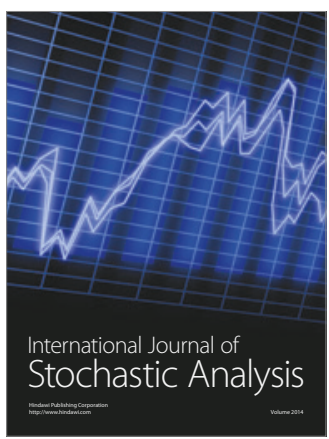

Probability and Statistics
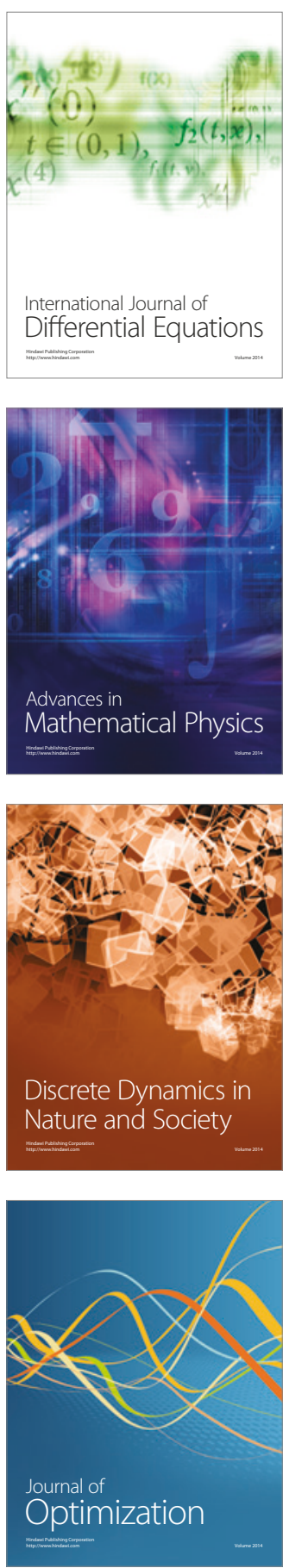